\title{
Two-Level Stability Analysis of Complex Circuits
}

\author{
Almudena Suárez, Fellow, IEEE, Franco Ramírez, Senior Member, IEEE
}

\begin{abstract}
A new methodology is proposed for the small- and large-signal stability analysis of complex microwave systems, containing multiple active blocks. It is based on a calculation of the system characteristic determinant that ensures that this determinant does not exhibit any poles in the right-hand side of the complex plane (RHS). This is achieved by partitioning the structure into simpler blocks that must be stable under either open-circuit (OC) or shortcircuit (SC) terminations. Thus, the system stability is evaluated by means of a two-level procedure. The first level is the use of pole-zero identification to define the $\mathrm{OC}-$ or SC-stable blocks, which, due to the limited block size, can be applied reliably. In large-signal operation, the $\mathrm{OC}$ - or SC-stable blocks are described in terms of their outer-tier conversion matrices. The second level is the calculation and analysis of the characteristic determinant of the complete system at the ports defined in the partition. The roots of the characteristic determinant define the stability properties. The Nyquist criterion can be applied since, by construction, the determinant cannot exhibit any poles in the RHS. In addition, one can use pole-zero identification to obtain the values of the determinant zeroes. Because the determinant is calculated at a limited number of ports, the analysis complexity is greatly reduced.
\end{abstract}

Index Terms-Stability analysis, Nyquist criterion, pole-zero identification, conversion-matrix approach.

\section{INTRODUCTION}

$\mathrm{D}$ ESIGNERS of nonlinear circuits often find qualitative differences between the solution simulated and the solution measured [1]-[5]. This is due, in most cases, to the instability of the solution simulated [6]-[10], which may give rise to undesired oscillations and other phenomena [11] [16]. The prediction of the stability properties is essential when using frequency-domain methods, such as an analysis based on the scattering parameters, in the case of a linear circuit, or harmonic balance (HB), in the case of a nonlinear one [2],[16][17]. This is because these methods only provide the steadystate solution and are unable to predict the reaction of this solution to the small perturbations that are always present in real life. Thus, complementary stability-analysis techniques are necessary. The mu factor and stability circles [18], used for the potential instability analysis of a two-port network, are limited to small-signal operation [19]-[22]. They can only be applied if the two-port network is stable when unloaded (Rollet proviso) [23], which must be verified with a performant stability analysis. In view of these limitations [19]-[22], other methods are used. There are two major types of rigorous stabilityanalysis methods: those based on the use of the Nyquist criterion

This work was supported in part by the Spanish Ministry of Science and Innovation and the European Regional Development Fund / Fondo Europeo de Desarrollo Regional (ERDF/FEDER) under research project TEC2017-88242-C3-1-R.
[2]-[3], [15], [20]-[22], [24]-[25] and those based on a polezero analysis [26]-[33].

The methods based on the Nyquist criterion [20]-[22], [24][25] enable a global evaluation of the solution stability in a single analysis, which simultaneously accounts for the whole circuit structure, without any problems of observability. However, the Nyquist criterion cannot be applied to complex functions $F(s)$ that are susceptible to exhibit both zeroes and poles in the right hand side of the complex plane (RHS) [24]. This is because the number $T$ of clockwise encirclements around the origin of the Nyquist plot $F(j \Omega)$, where $\Omega$ is the imaginary part of $s$, provides the difference between the number $Z$ of RHS zeroes of $F(s)$ and the number $P$ of RHS poles of $F(s)$, that is, $T=Z-P$ [20]-[22], [24]-[25]. If the chosen function $F(s)$ can exhibit both RHS zeroes (agreeing with the characteristic roots that define the solution stability properties) and RHS poles, there can be a wrong prediction of the stability properties, due to the subtraction of $P$. Obtaining a function that cannot exhibit both RHS zeroes and RHS poles is not an easy task. For instance, an ordinary impedance or admittance function, calculated at a given circuit node or branch, can have both $Z \neq 0$ and $P \neq 0$ [24]. This is because the derivatives of the intrinsic nonlinearities with respect to their control variables (responsible for the circuit activity) affect both the numerator and denominator. In contrast, the characteristic determinant calculated from the perturbed HB formulation [2], [16], [25] cannot exhibit RHS poles. This is because the Jacobian matrix of the intrinsic nonlinearities only affects the numerator of the determinant. Thus, the poles of this determinant can only come from the passive linear elements and are necessarily located in the left-hand side of the complex plane (LHS). RHS poles are not possible either in the normalized determinant function defined in [20]-[22]. This is obtained through the calculation of a sequence of open-loop transfer functions (one per active element) or, more recently, from an admittance representation of the network at the nodes of the nonlinear elements susceptible to give rise to instability. The method is accurate and complete when having access to the device intrinsic terminals. It is less reliable when the devices are described with black-box models containing linear and nonlinear elements.

Unlike the methods based on the use of the Nyquist criterion, the pole-zero analysis [26]-[32] is applied to a closed-loop transfer function that can contain both RHS poles and RHS

A. Suárez and F. Ramírez are with the Communications Engineering Department, University of Cantabria, 39005, Santander, Spain (e-mail: suareza@unican.es; ramirezf@unican.es). 
zeroes. This is because the poles and zeroes are distinctly detected, which in the identification method is achieved by fitting the transfer function with a quotient of polynomials. The pole-zero analysis [26]-[32] relies on the fact that all the transfer functions that can be defined in a linear system share the same denominator and therefore should exhibit the same poles [26]-[31]. These poles agree with the roots of the characteristic determinant that define the stability properties. However, unlike the poles, the zeroes depend on the particular transfer function and cancellations/quasi-cancellations of RHS poles and RHS zeroes may take place [30]. If the quasicancelled poles are invalid (due, for instance, to a too high identification order), one may believe that the circuit is unstable, when it is stable. However, the quasi-cancelled poles may also be due to a low observability of an existing instability. The recent method [32], based on projecting the closed-loop transfer function on an orthogonal basis of stable and unstable functions, prevents artificial quasi-cancellations that can result from the fitting with a rational function. However, this method can still miss instabilities due to a low observability. As stated in [32], the chance of missing instabilities is reduced through a sequential analysis of many different transfer functions. This may become demanding in complex multi-device structures [33].

Here a two-level methodology for the stability analysis of complex systems is proposed, which formalizes an idea used in [34]-[35] for the small-signal stability analysis of non-Foster networks [36]-[37]. In addition, it addresses the significantly more complex case of the large-signal stability analysis. The new method relies on the calculation of the system characteristic determinant, thus coping with the observability limitations of the closed-loop stability analysis. The determinant is calculated in a manner that ensures that it does not exhibit any poles in the RHS. This is achieved by partitioning the system into simpler active blocks that are stable under either open-circuit (OC) or short-circuit (SC) terminations, connected through a passive linear network. In large-signal operation, the $\mathrm{OC}-$ or SC-stable blocks are described in terms of their outer-tier conversion matrices. Note that even if the composing blocks are stable, the complete system may be unstable [24], [38]-[45], due to feedback effects and/or variations in the loading conditions resulting from the block interactions. Thus, the method consists of a two-level procedure. The first level is the use of pole-zero identification [26]-[31] to define the OC- or SC-stable blocks, which, due to the limited block size can be applied reliably, without observability problems. The second level is the calculation and analysis of the characteristic determinant of the complete system at the reference ports defined in the partition. The use of a small number of ports facilitates the extension to the large-signal stability analysis.

The paper is organized as follows. Section II presents the methodology for the small-signal stability analysis. Section III presents the methodology for the large-signal stability analysis.

\section{Small-Signal Stability ANAlysis}

The new analysis method is based on partitioning the system into simpler active blocks that are stable under either open-circuit or short-circuit terminations. Note that the method does not impose the stability of the blocks that are connected together. Instead, what the method requires is a (virtual) decomposition of the system into (other) blocks that are stable under either OC or SC terminations. As will be shown, the number of analysis ports associated to each block can be increased to facilitate the fulfilment of the OC or SC stability.

\section{A. Open-circuit and short-circuit stability}

In a driving-point impedance function [38]-[41], the excitation corresponds to a small-signal current $I(s)$ and the response is a voltage $V(s)$ :

$$
Z(s)=\frac{V(s)}{I(s)}=\frac{p(s)}{q(s)}
$$

where $Z(s)$ is the transfer function and $p(s)$ and $q(s)$ are, respectively, the polynomials of the numerator and denominator. It is assumed that in (1) there are no cancellations of RHS poles and RHS zeroes, due to hidden instabilities. In the absence of the current source, $I(s)=0$ [open-circuit (OC) termination], one will have the following characteristic equation [38]:

$$
q(s) V(s)=0
$$

where $q(s)$ is the characteristic polynomial. The stability of the block itself (in the absence of input sources) is defined by the roots of $q(s)$, or characteristic roots. Comparing (1) and (2), under an OC termination, these roots agree with the poles of the transfer function in (1). If all the characteristic roots are in the LHS, the analyzed block is OC-stable.

Now, a system composed of two blocks (Block 1 and Block 2 ), in the absence of input sources, will be considered. The blocks are connected as shown in Fig. 1 and are assumed to be opencircuit stable (OC-stable) [Fig. 1(a)]. In terms of the common branch current, one can write the following characteristic equation:

$$
\left[Z_{1}(s)+Z_{2}(s)\right] I(s)=Z_{T}(s) I(s)=F(s) I(s)=0
$$

In this one-dimensional case, $F(s)$ is conceptually equivalent to the characteristic determinant. Because Block $\mathrm{k}_{1}$ and Block $_{2}$ are OC-stable, neither $Z_{1}$ nor $Z_{2}$ can have any poles in the RHS. Representing $Z_{1}$ and $Z_{2}$ in a manner analogous to (1), one can express $F(s)$ as:

$$
\begin{aligned}
F(s) & =Z_{1}(s)+Z_{2}(s)=\frac{p_{1}(s)}{q_{1}(s)}+\frac{p_{2}(s)}{q_{2}(s)} \\
& =\frac{p_{1}(s) q_{2}(s)+p_{2}(s) q_{1}(s)}{q_{1}(s) q_{2}(s)}
\end{aligned}
$$

The stability properties of the two-block system are defined by the zeroes of $F(s)$. In agreement with the derivations in [38]-[41], the function $F(s)$ cannot have any RHS poles because neither $q_{1}(s)$ nor $q_{2}(s)$ can exhibit any RHS roots, since the respective blocks are $\mathrm{OC}-$ stable. Thus, the function $F(s)$ will not suffer from any uncertainties associated with the possible cancellations or quasi-cancellations of RHS zeroes and poles. 


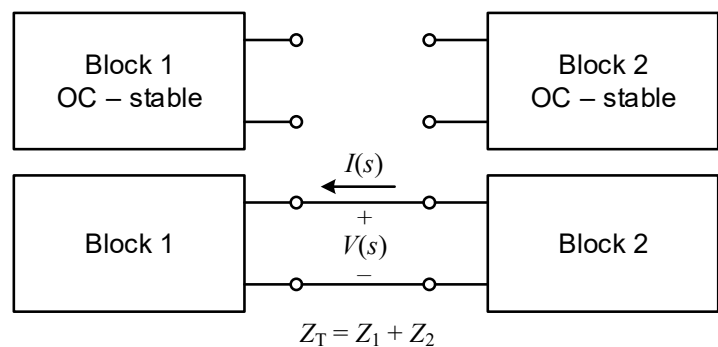

(a)

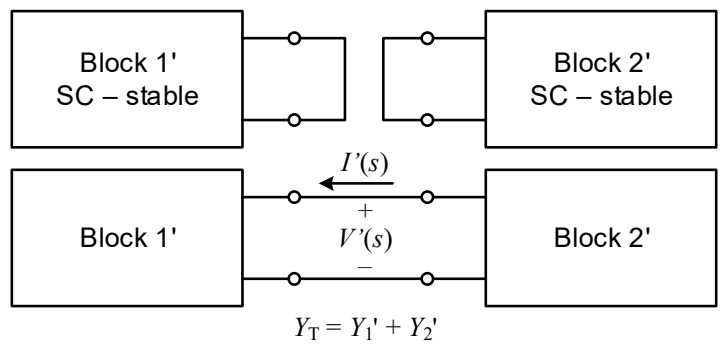

(b)

Fig. 1. Open and short-circuit stability. (a) OC-stable blocks. Analysis in terms of impedances. (b) SC-stable blocks. Analysis in terms of admittances.

Now, the perturbation behavior of the same system of two OC-stable blocks will be analyzed in terms of the common node voltage $V(s)$. The characteristic equation is:

$$
\left[Y_{1}(s)+Y_{2}(s)\right] V(s)=F_{p}(s) V(s)=0
$$

where the admittance functions $Y_{1}(s), Y_{2}(s)$ are the inverses of $Z_{1}(s), Z_{2}(s)$. Taking this into account, one obtains:

$$
\begin{aligned}
F_{p}(s) & =Y_{1}(s)+Y_{2}(s)=\frac{q_{1}(s)}{p_{1}(s)}+\frac{q_{2}(s)}{p_{2}(s)} \\
& =\frac{p_{1}(s) q_{2}(s)+p_{2}(s) q_{1}(s)}{p_{1}(s) p_{2}(s)}
\end{aligned}
$$

The physical system is the same in the two cases, so, as expected, the zeroes of $F_{p}(s)$ (defining the system stability), agree with those of (4). However, unlike the situation in (4), the function $F_{p}(s)$ can exhibit RHS poles, coming from the zeroes of $p_{1}(s) p_{2}(s)$, or, equivalently, the zeroes of the OC-stable transfer functions $\left(Z_{1}, Z_{2}\right)$ of the individual blocks.

For the dual case of two stable blocks under a short-circuit termination (SC-stable), we will consider two other blocks (Block ${ }_{1}^{\prime}$ and Block ${ }_{2}{ }^{\prime}$ ), shown in Fig. 1(b). Because these two blocks are SC-stable, their corresponding admittance functions $Y_{1}^{\prime}(s), Y_{2}{ }^{\prime}(s)$ do not have any RHS poles. Expressing the characteristic system in terms of the common-node voltage, the stability properties are defined by the zeroes of the following function, agreeing with the characteristic roots:

$$
\begin{aligned}
F^{\prime}(s) & =Y_{1}{ }^{\prime}(s)+Y_{2}{ }^{\prime}(s)=\frac{p_{1}{ }^{\prime}(s)}{q_{1}{ }^{\prime}(s)}+\frac{p_{2}{ }^{\prime}(s)}{q_{2}{ }^{\prime}(s)} \\
& =\frac{p_{1}{ }^{\prime}(s) q_{2}{ }^{\prime}(s)+p_{2}{ }^{\prime}(s) q_{1}{ }^{\prime}(s)}{q_{1}{ }^{\prime}(s) q_{2}{ }^{\prime}(s)}
\end{aligned}
$$

In a manner analogous to (4), the function $F^{\prime}(s)$ cannot exhibit any RHS poles, so it will not suffer from uncertainties associated with the possible cancellations or quasicancellations of RHS zeroes and poles.

In summary, to avoid the possible coexistence of RHS zeroes and RHS poles, OC-stable blocks should be represented in terms of impedances and SC-stable blocks should be represented in terms of admittances. On the other hand, a system composed of OC-stable blocks must be analyzed in terms of the branch currents and a system composed of SC-stable blocks must be analyzed in terms of the node voltages.

\section{B. New analysis methodology}

To generalize the analysis, a system with $N$ active blocks (containing both linear and nonlinear elements), interconnected through a purely passive linear network, will be considered. For an insightful derivation, the simplest case of $N$ one-port active blocks will be initially considered, as in the case of circuits coupled at their output ports. In general, depending on the block connections, two or more ports per active block may be necessary, and this multi-port case is treated later in this section. Initially, it will be assumed that the $N$ one-port active blocks are OC-stable, so they will be described with their corresponding impedance functions $Z_{A, n}$, where $n=1 \ldots N$. In turn, the passive linear network will be described with its $N \times N$ impedance matrix $\left[Z_{P}\right]$.

One must guarantee that there are no hidden instabilities in the blocks defined by $Z_{A, n}$. With this aim, the OC-stability of each active block will be evaluated inside the block through pole-zero identification applied to a transfer function defined at a sensitive location (device terminals), ensuring observability. As usual, the stable behavior must be confirmed by repeating the analysis at different locations [26]-[31]. If the OC block is stable, none of its associated transfer functions can exhibit RHS poles (and this will also be the case for $Z_{A, n}$ ). Considering, as stated, one-port active blocks, the characteristic system is:

$$
\left\{\begin{array}{l}
\left.\left[\begin{array}{cccc}
Z_{A, 1}(s) & 0 & \ldots & 0 \\
0 & Z_{A, 2}(s) & \ldots & 0 \\
\vdots & \vdots & \ddots & \vdots \\
0 & 0 & \ldots & Z_{A, N}(s)
\end{array}\right]+\right\} \\
{\left[\begin{array}{ccc}
Z_{P, 11}(s) & \ldots & Z_{P, 1 N}(s) \\
\vdots & \vdots & \vdots \\
Z_{P, N 1}(s) & \ldots & Z_{P, N N}(s)
\end{array}\right]}
\end{array}\right\}\left[\begin{array}{c}
\Delta I_{1} \\
\vdots \\
\Delta I_{N}
\end{array}\right]=\left[\begin{array}{c}
0 \\
\vdots \\
0
\end{array}\right]
$$

where $s$ is the perturbation frequency and $\Delta I_{1}$ to $\Delta I_{N}$ are the current increments at the connection branches. Note that (8) must respect the actual connection between the system blocks, that is, it must be a valid representation of the perturbed system (without eliminating any elements). The stability properties are defined by the roots of the characteristic determinant:

$\operatorname{det}\left\{\left[\begin{array}{cccc}Z_{A, 1}(s) & 0 & \ldots & 0 \\ 0 & Z_{A, 2}(s) & \ldots & 0 \\ \vdots & \vdots & \ddots & \vdots \\ 0 & 0 & \ldots & Z_{A, N}(s)\end{array}\right]+\left[\begin{array}{ccc}Z_{P, 11}(s) & \ldots & Z_{P, 1 N}(s) \\ \vdots & \vdots & \vdots \\ Z_{P, N 1}(s) & \ldots & Z_{P, N N}(s)\end{array}\right]\right\}=0$ 
Obviously, under the OC-stability condition imposed to the $N$ active blocks, the characteristic determinant (9) cannot exhibit any RHS poles, so the Nyquist criterion can be applied reliably. In some cases, the complexity of the Nyquist plot can be reduced multiplying (8) by the inverse of the passive linear matrix $\left[Z_{P}(s)\right]$, which cannot introduce any RHS poles. Because the active blocks are stable (with no possibility of cancellation of RHS zeroes and with RHS poles), any instability resulting from the block connection should be detectable through (9). In case the blocks are $\mathrm{SC}-$-stable, the characteristic system should be written in terms of admittance matrices:

$$
\left\{\begin{array}{l}
\left\{\begin{array}{cccc}
Y_{A, 1}(s) & 0 & \ldots & 0 \\
0 & Y_{A, 2}(s) & \ldots & 0 \\
\vdots & \vdots & \ddots & \vdots \\
0 & 0 & \ldots & Y_{A, N}(s)
\end{array}\right]+ \\
{\left[\begin{array}{ccc}
Y_{P, 11}(s) & \ldots & Y_{P, 1 N}(s) \\
\vdots & \vdots & \vdots \\
Y_{P, N 1}(s) & \ldots & Y_{P, N N}(s)
\end{array}\right]}
\end{array}\right\}\left[\begin{array}{c}
\Delta V_{1} \\
\vdots \\
\Delta V_{N}
\end{array}\right]=\left[\begin{array}{c}
0 \\
\vdots \\
0
\end{array}\right]
$$

where $Y_{A, n}$, where $n=1 \ldots N$ are the admittance functions of the SC-stable active blocks, which compose a diagonal matrix, $\left[Y_{P}(s)\right]$ is the admittance matrix describing the purely passive linear network (we assume that this matrix is defined), and $\Delta V_{1}$ to $\Delta V_{N}$ are the voltage increments at the block ports. As in the case of (8), (10) must respect the actual connection between the system blocks, that is, it must be a valid representation of this system (without short-circuiting any elements). As in the previous case, the SC-stability is evaluated inside each block through pole-zero identification applied to a transfer function defined at a sensitive location, ensuring observability. Under the SC-stability condition imposed to the active blocks, the characteristic determinant associated with (10) cannot exhibit any RHS poles, so the Nyquist criterion can be applied reliably.

The two systems (8) and (10) can be extended to the case of active blocks described with two-port networks (or, in general, $L$-port networks). In the case of OC-stable active blocks, impedance elements $Z_{A, n}$ in (8) will become matrices. The dimension of the passive linear matrix $\left[Z_{p}\right]$ will increase to accommodate the extra ports, but the analysis procedure will be analogous. An analogous procedure would also be applied in the case of the admittance-type analysis in (10). The extension of the formulation to a system containing both OC-stable and SCstable blocks is possible, though it will rarely be needed.

Regarding the guarantee of existence of a system decomposition into $\mathrm{OC} / \mathrm{SC}$ stable blocks, note that by defining the active-block ports in (10) at the intrinsic device terminals one would obtain the formulation in [2], [16], [25], providing a characteristic determinant that, by construction, cannot exhibit any RHS poles. Departing from the above limit case, one can argue that the OC/SC stability condition will be fulfilled by most transistors (including their extrinsic elements) at their external terminals, since it agrees with the Rollet proviso that enables the application of the widely-spread (though limited) Rollet criteria. On the other hand, it is highly unlikely that all the transistors become OC/SC unstable when adding some passive linear elements. Regarding the procedure for the decomposition, one should initially consider the ports of the active subcircuits responsible for particular functions, such as amplification, frequency multiplication etc... Particularizing, for instance, to an impedance analysis, if some of these subcircuits are OC unstable, one should define additional ports in these subcircuits. An example is presented in the following.

The method has been applied to the small-signal stability analysis of a system composed by three Class-E power amplifiers (based on an ATF50189 transistor) under output coupling effects [see Fig. 2(a)]. In driven conditions, and when isolated from the system, the amplifier reaches a peak efficiency of $60 \%$ with an output power of $24 \mathrm{dBm}$. The coupled system in Fig. 2(a) can be representative of instability mechanisms due to output mismatch [46]-[49] and enables a reliable validation of the new analysis method with pole-zero identification. In a practical application, the output coupling may be due to antenna cross talk, intensively investigated these days due to its impact on MIMO systems [50]-[51]. Here, the aim is to implement a reliable demonstrator of the new mathematical procedure, instead of obtaining a model/description of a physical cross-talk effect. Thus, a versatile coupling network containing a $\lambda / 4$ transmission line at $800 \mathrm{MHz}$, bounded by inductors and resistors, is considered, with the initial values $R=5 \Omega$ and $L=4.7 \mathrm{nH}$.

The three amplifiers in Fig. 2(a) are identical. However, for the small-signal stability analysis, series feedback, in the form of a parallel L-C network, has been introduced in the third amplifier [Fig. 2(c)]. This $\mathrm{L}-\mathrm{C}$ network is purposely introduced to induce an instability. Initially, the system is partitioned into three active blocks (each corresponding to one amplifier under a single OC termination at the output port), plus the coupling network (Fig. 2). The stability is analyzed versus the gate-bias voltage $V_{G S}$, which is very convenient to test the method capabilities, since one can expect qualitative stability changes versus this parameter. This is because the oscillations are quenched when decreasing $V_{G S}$ towards the conduction threshold.

The stability analysis of the amplifier blocks is carried out through pole-zero identification [26]-[31]. For this analysis, a small-signal test current $I_{s}$ at the perturbation frequency $\Omega$ is introduced in parallel at a sensitive circuit node. Then, a closedloop transfer function is defined as the ratio between the gatenode voltage and the test current [26]-[31], doing: $Z_{s}(\Omega)=V_{s}(\Omega) / I_{s}$. Under sufficient observability, one can obtain the poles from any transfer function, since they all share the same denominator, agreeing with the characteristic determinant. In practical circuits, the number of poles can be very high. However, most of the poles will be in the LHS, far from the imaginary axis. Only a few poles will be critical or near critical, and these are the ones with the largest real parts (dominant poles). 


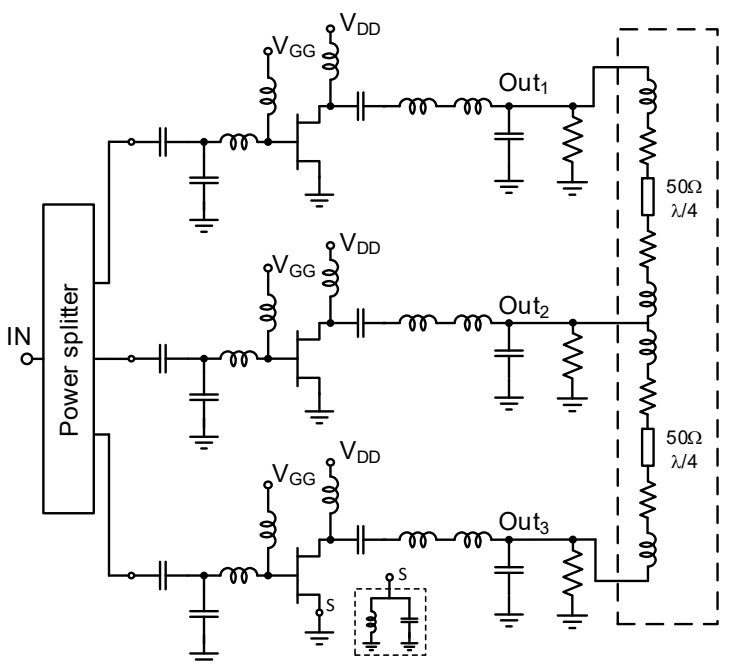

(a)

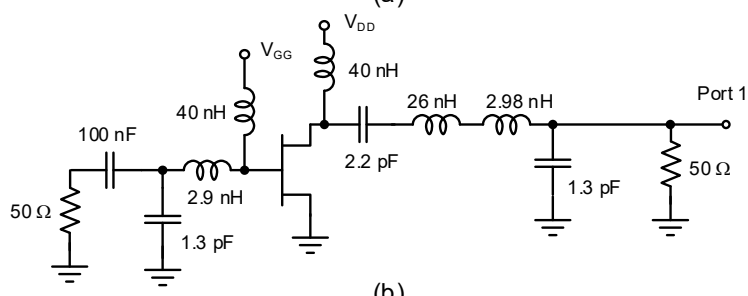

(b)

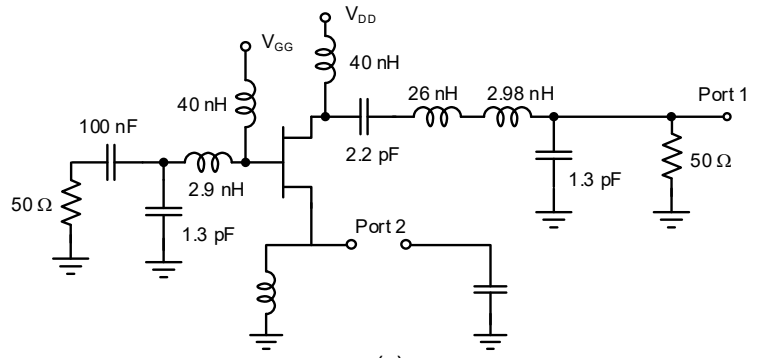

(c)

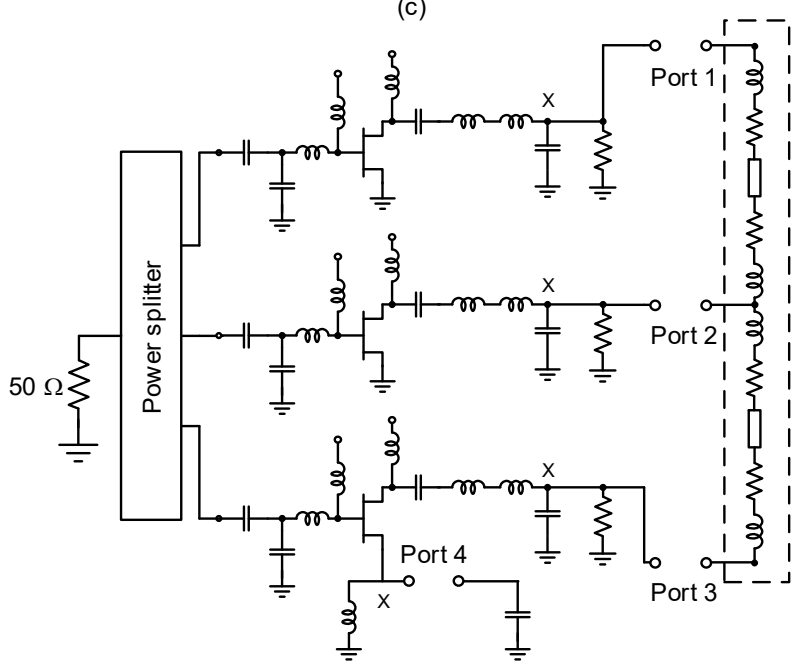

(d)

Fig. 2. Definition of blocks in a system of three power amplifiers under output coupling effects. They are Class-E power amplifiers based on an ATF50189 transistor. (a) Schematic of the full system. (b) Block representation of the first and second amplifiers. They are one-port OC-stable blocks. (c) Block representation of the third amplifier, with a series-feedback network. It is a twoport OC-stable block. (d) Complete structure with four analysis ports to be analyzed with (9). Alternatively, the analysis based on the characteristic determinant of the admittance system (10) is applicable at the ports defined between the nodes " $x$ " and ground.
In each of the amplifier blocks, the test current $I_{s}$ is introduced in parallel at the gate terminal. Fig. 3(a) presents the variation of the real part of the dominant poles and zeroes of the first and second amplifiers versus $V_{G S}$. The poles are indicated with " $x$ " and the zeroes are indicated with "o". Because the two amplifiers are identical in standalone operation, only one has been analyzed. As seen in Fig. 3(a), there is a fast variation of the poles and zeroes near the conduction threshold. In this case, the dominant poles are a pair of complex-conjugate poles, so when traced versus the parameter $\left(V_{G S}\right)$, the real part is the same for both. For $V_{G S}$ below (above) the conduction threshold, the identification also detects one pair (two pairs) of dominant complex-conjugate zeroes, with no influence on the stability properties. The real part of the dominant (complex-conjugate) poles is always negative, so the amplifier is stable for all the $V_{G S}$ values. The same conclusion is obtained when the test current is introduced at other device terminals. Thus, the first and second amplifiers are stable under an output $\mathrm{OC}$ termination at the port indicated in Fig. 2(b).
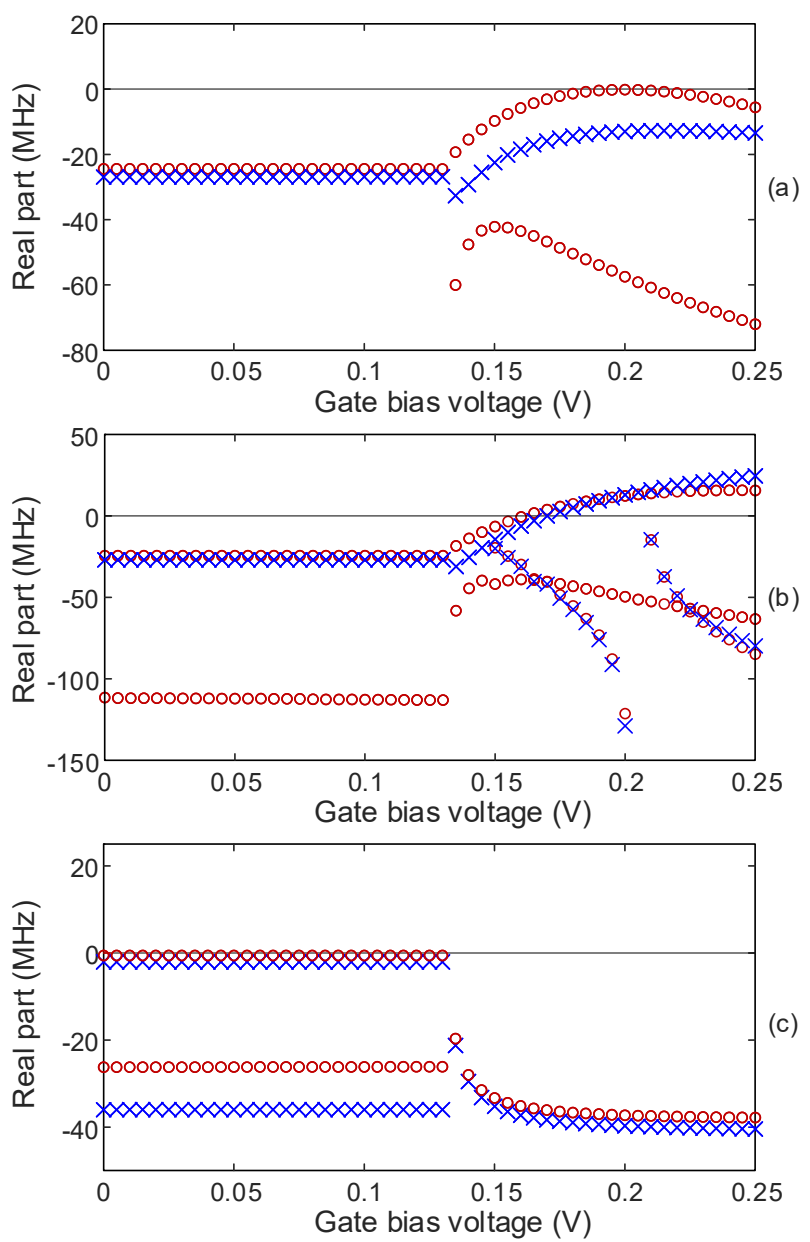

Fig. 3. Small-signal analysis. Open-circuit stability of the individual blocks in the system of Fig. 2(a), analyzed with pole-zero identification. Variation of the real part of the dominant poles $(\times)$ and zeroes (o) versus $V_{G S}$. The stability information is in the poles. The zeroes have no impact on the stability properties. (a) First and second (identical) amplifiers. Only one is analyzed. It is stable for all the $V_{G S}$ values. (b) Third amplifier [in Fig. 2(c)] in OC conditions at Port 1 only. It becomes unstable at $V_{G S}=0.16 \mathrm{~V}$. (b) Third amplifier in OC conditions at the two ports defined in Fig. 2(c). It is stable for all the $V_{G S}$ values. 
When analyzing the stability of the third amplifier (including the purposely-introduced series feedback) under a single OC termination at the output port, one obtains the results shown in Fig. 3(b), where the real part of its dominant poles (and zeroes) has been represented versus $V_{G S}$. The real part of a pair of complex-conjugate poles becomes positive at $V_{G S}=0.16 \mathrm{~V}$, so the amplifier in Fig. 2(c) is unstable for $V_{G S}>0.16 \mathrm{~V}$. To obtain an OC-stable block associated with the third amplifier, we will define a second port, as shown in Fig. 2(c). This second port breaks the connection of the source terminal to the capacitor of the $\mathrm{L}-\mathrm{C}$ feedback network. Note that the removed capacitor will be absorbed in the passive linear impedance matrix $\left[Z_{P}\right]$ when applying (9). With the two ports defined in Fig. 2(c) in opencircuit conditions, the third amplifier is OC-stable for all the $V_{G S}$ values. The resulting variation of the real part of the dominant poles versus $V_{G S}$ is shown in Fig. 3(c).

With the new procedure, the characteristic determinant is obtained calculating the determinant of the total impedance matrix at the four ports indicated in Fig. 2(d). This is consistent with our definition of the OC-stable active blocks and with the impedance-matrix formalism (8) and (9). Note that the capacitor that had been removed from the third amplifier is included in the passive linear network described with the matrix $\left[Z_{P}\right]$.

Fig. 4(a) presents the Nyquist plots obtained for two different values of the gate-bias voltage $\left(V_{G S}\right)$, simultaneously varied in the three amplifiers, before and after a primary Hopf bifurcation [1]-[5], [52] that takes place at $V_{G S}=0.165$ V. Fig. 4(b) presents an expanded view about the origin of this plot. For $V_{G S}=0.16 \mathrm{~V}$, the plot (indicated with a label) does not encircle the origin, so it predicts a stable behavior. In contrast, for $V_{G S}=0.17 \mathrm{~V}$ (indicated with a label) the plot encircles the origin in the clockwise sense, so it predicts unstable behavior.

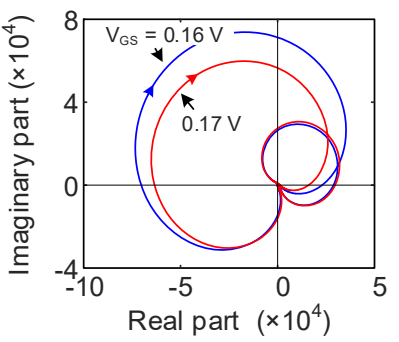

(a)

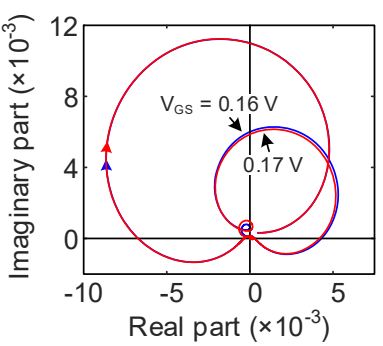

(c)

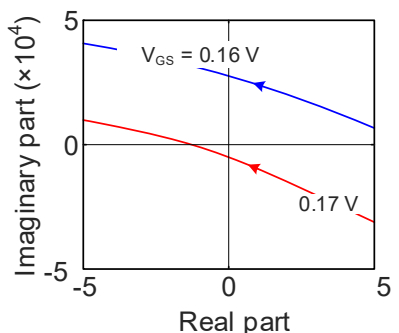

(b)

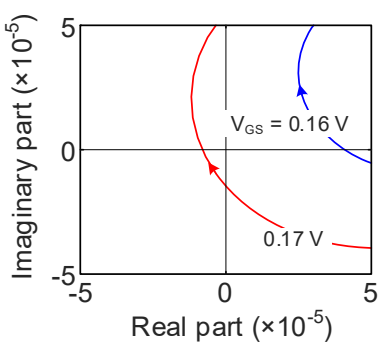

(d)
Fig. 4. Small-signal stability for two different gate-bias voltages, indicated with labels. (a) Nyquist analysis of the characteristic determinant (9), obtained by calculating the total impedance matrix at the four ports shown in Fig. 2(d). Nyquist plots for $V_{G S}=0.16 \mathrm{~V}$ (stable) and $V_{G S}=0.17 \mathrm{~V}$ (unstable). (b) Expanded view about the origin. (c) Analysis based on the characteristic determinant of the admittance system (10). The total admittance matrix is calculated at the ports defined between each node " $x$ " and ground. (d) Expanded view about the origin.
To show the flexibility in the choice of the analysis ports, an alternative analysis based on the characteristic determinant of the admittance system (10) has also been performed. This analysis requires $\mathrm{SC}-$ stable active blocks. When the node(s) denoted with an " $x$ " in each amplifier [Fig. 2(d)] are shortcircuited to ground, the newly defined active blocks are stable. Then, the stability of the whole structure can be analyzed using the total admittance matrix calculated at the four ports defined between each node " $x$ " and ground. The Nyquist plots obtained for the same two values of gate-source voltage $\left(V_{G S}=0.16 \mathrm{~V}\right.$ and $V_{G S}=0.17 \mathrm{~V}$ ) are shown in Fig. 4(c) and Fig. 4(d). As expected, the stability predictions are the same as those obtained with (9).

In addition, pole-zero identification has been applied to the characteristic determinant (9), which provides the results shown in Fig. 5. Note that the stability information is now in the zeroes of the determinant, agreeing with the characteristic roots. Again, the poles are indicated with " $\times$ " and the zeroes are indicated with "o". Fig. 5(a) presents the variation versus $V_{G S}$ of the dominant zeroes and poles of the determinant in the plane defined by the real and imaginary parts. All the determinant poles (with no influence on the stability properties) are quite far from the dominant determinant zeroes (characteristic roots). This is more evident in Fig. 5(b), which presents the variation of the real part of the dominant zeroes and poles versus $V_{G S}$. The change in the configuration of the zeroes occurs when the transistors turns on. As gathered from Fig. 5, the system is unstable from the Hopf bifurcation [14]-[15] occurring at $V_{G S}=0.165 \mathrm{~V}$. We emphasize that, by construction, the determinant cannot have any RHS poles, which avoids the problem of zero-pole cancellations or quasi-cancellations. The zeroes obtained from the identification of the characteristic determinant of the admittance-based system (10) are overlapped.

For comparison, Fig. 6 presents the results of conventional pole-zero identification applied to a closed-loop transfer function. Three different functions have been considered for this analysis, calculated by introducing a test current $I_{s}$ in parallel at a given node and obtaining the ratio between the node voltage $V_{s}$ and $I_{s}$ [26]-[31]. Fig. 6(a) presents the variation of the real part of the dominant poles and zeroes versus $V_{G S}$ when the test current $I_{n}$ is connected at the gate terminal of the transistor in the first amplifier. The poles (providing the stability information) are quasi-cancelled with zeroes, which is due to the limited observability. Fig. 6(b) presents the variation of the real part of the dominant poles and zeroes when the test current is connected at the gate terminal of the third amplifier (responsible for the instability). As expected, the poles agree with the zeroes of the characteristic determinant. However, in Fig. 6(b) the RHS poles have nearby zeroes. This coexistence of RHS zeroes and poles is impossible with the new method. Finally, Fig. 6(c) presents the variation of the real part of the dominant poles and zeroes when the test current is introduced at the node where the third amplifier (responsible for the instability) is connected to the coupling network. The RHS poles are again quasi-cancelled with RHS zeroes. 

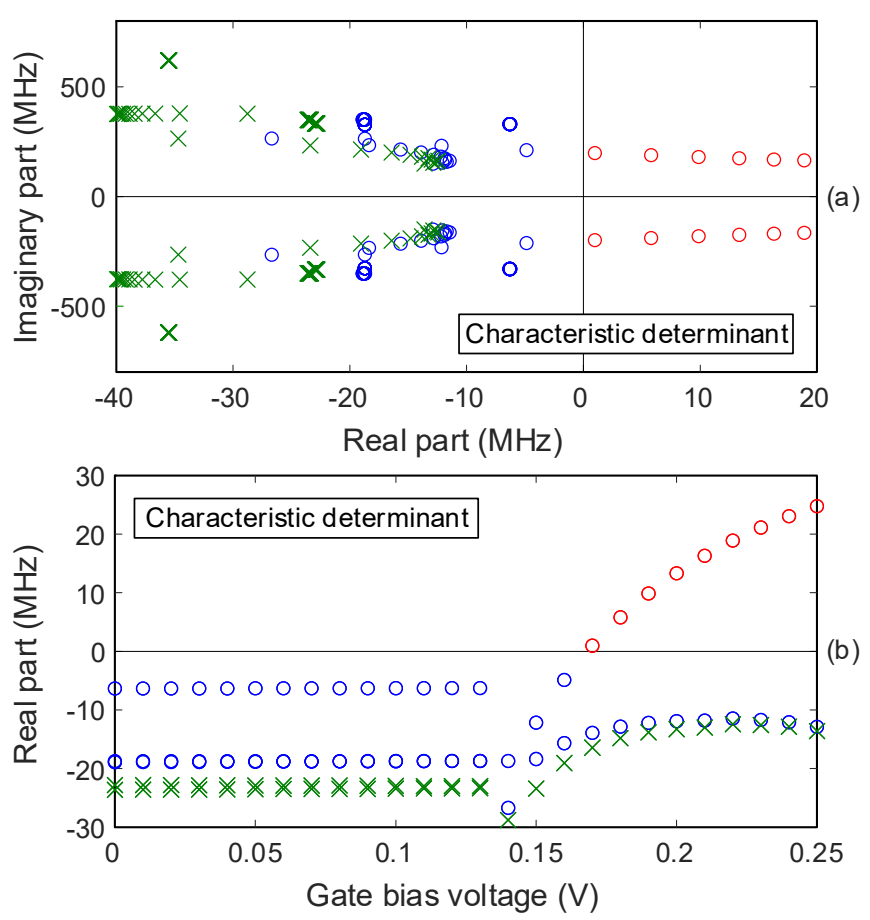

Fig. 5. Small-signal analysis. Identification of the characteristic determinant (9) . Note that the stability information is now in the zeroes of the determinant, agreeing with the characteristic roots. By construction, the determinant cannot exhibit any RHS poles. (a) Variation of the zeroes (o) and poles $(\times)$ versus $V_{G S}$, in the plane defined by the real and imaginary parts. (b) Variation of the real part of the dominant zeroes and poles versus $V_{G S}$.

As has been shown, the characteristic determinant can be analyzed through both the Nyquist criterion and pole-zero identification applied to (9)-(10). The possibility to use the two methods is interesting since they can be considered as complementary. In the Nyquist criterion the perturbation frequency is ideally swept from 0 to $\infty$ (in practice, beyond the highest $f_{\max }$ of the transistors [20]-[22]), which is done at once, in a single sweep. Instead, pole-zero identification requires a partition of the frequency band into elementary bands [26]-[31] to ensure a sufficient accuracy of the least-squares identification algorithm. On the other hand, the Nyquist plot can be intricate (and difficult to interpret), whereas pole-zero identification directly provides the zeroes of the characteristic determinant.

\section{LARGE-Signal StabiLITy ANALYSIS}

The large-signal stability analysis will be applied to a periodic steady-state solution obtained with harmonic balance. Then, the circuit will be linearized about this solution with the conversion-matrix approach, to obtain the stability information. Section III.A presents a formal derivation of the characteristic determinant and demonstrates that it cannot exhibit RHS poles. Section III.B describes the practical calculation of the determinant from large-signal small-signal simulations in commercial software. Section III.C presents an application example.
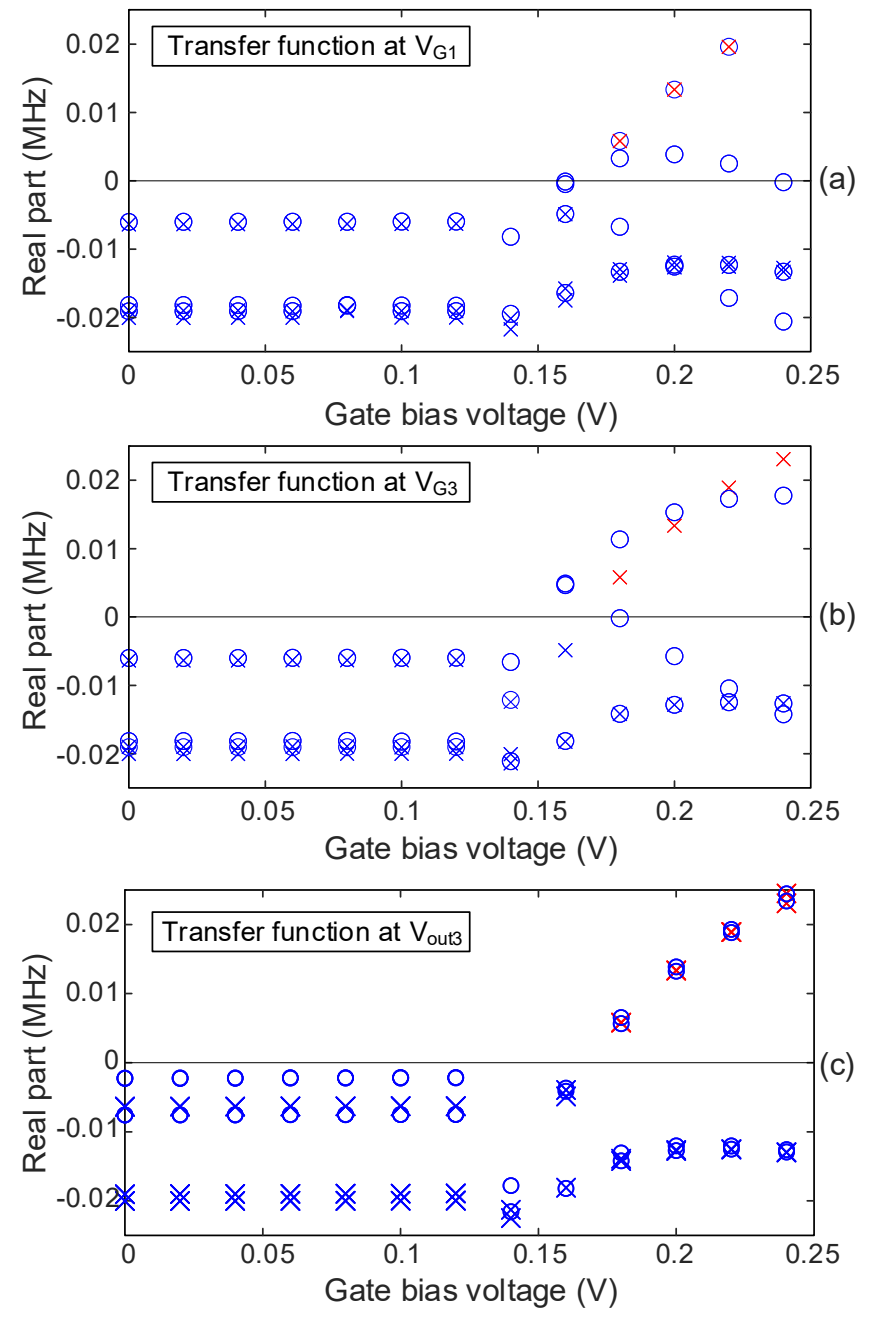

Fig. 6. Small-signal stability analysis based on the pole-zero identification of a transfer function. The stability information is in the poles. The real part of the poles ( $\times$ ) and zeroes (o) is represented versus $V_{G S}$. (a) Test current at the gate terminal of the transistor in the first amplifier. (b) Test current at the gate terminal of the third amplifier (responsible for the instability). (c) Test current at the node where the third amplifier is connected to the coupling network. The unstable poles are quasi-cancelled with RHS zeroes.

\section{A. Formulation}

In the presence of a small-amplitude perturbation, there will be a mixing between the frequencies of the periodic solution $j m \omega_{o}$, where $\omega_{o}$ is the fundamental frequency and $-M \leq m \leq M$, and the complex frequency $s$ (associated with the perturbation), which will give rise to the sidebands: $j m \omega_{o}+s$ [2], [10], [16], [25]. For an insightful exposition of the analysis method, a system composed by one-port active blocks that are either OCstable or SC-stable is considered. As in the small-signal case, the generalization to multi-port blocks is straightforward and the extension to blocks that are OC stable at some ports and SC stable at the other ports is possible, but will rarely be needed.

Each active block will be represented with an outer-tier conversion matrix of either impedance or admittance type. The choice will depend on the stability properties of the active blocks under open-circuit (OC) and short-circuit (SC) terminations. The particular configuration of the blocks will give a hint on the convenience of either OC or SC stability. For 
instance, in the case of Fig. 2(a), if the amplifiers are stable on their own, the OC-stability condition will be easily satisfied. Note that the stability verification (under either OC or SC) must be carried out linearizing the blocks about the periodic steadystate solution of the complete system, in which all the blocks are connected. This solution is given by the full set of voltage and current values at the harmonic terms $m \omega_{o}$, where $m$ goes from $-M$ to $M$.

Fig. 7 illustrates the procedure to analyze the $\mathrm{OC}$ or $\mathrm{SC}$ stability of the active blocks, showing the test current introduced for the pole-zero identification. The OC-stability test of a given block is carried out terminating all the sideband frequencies $m \omega_{o}+\Omega$ in open circuits, as sketched in Fig. 7(a). This opencircuit termination should not affect the harmonic loads at $m \omega_{o}$, which is implemented through an ideal filter that is a short circuit at $m \omega_{o}$ and open circuit at the sideband frequencies $m \omega_{0}+\Omega$ [Fig. 7(a)]. In turn, the SC-stability test of a given block [Fig. 7(b)] is carried out terminating all the sideband frequencies $m \omega_{o}+\Omega$ in short circuits. This termination should not affect the harmonic loads at $m \omega_{o}$, which is achieved using the ideal filter shown in Fig. 7(b). One should emphasize that the short-circuit termination should respect the block topology, that is, it must not short circuit any elements of the block itself.

As stated, the OC or SC terminations disconnect the blocks at the sideband frequencies only; they are fully connected at the steady-state solution at $m \omega_{0}$, where $-M \leq m \leq M$. The OC or SC large-signal stability analysis of the composing blocks is carried out through pole-zero identification applied in a conventional manner [26]-[31]. That is, a small-signal test current $I_{s}$ at the frequency $\Omega$ (incommensurable with $\omega_{o}$ ) is connected in parallel at a sensitive node of the block (in either OC or SC conditions at the sidebands). Then, the conversion-matrix approach is used to obtain a transfer function defined as the ratio between the node voltage $V_{s}$ at $\Omega$ and $I_{s}$. (The results must be validated repeating the analysis at several locations). If the stability condition is not fulfilled defining a one-port network, one can increase the number of ports, as done in Section II. This will also be needed if the block is connected to the system through more than one port.

The case of a system composed by $N$ blocks that are OCstable will be initially considered. For the calculation of the characteristic determinant, each block $n$ will be described with an impedance-type outer-tier conversion matrix. The blocks are connected to the system at the steady-state frequencies $m \omega_{o}$, but in OC conditions at the sidebands. This way the block nonlinearities are in the same operation conditions as in the full system, with identical frequency-conversion effects.

Fig. 8 illustrates the procedure to calculate the impedance/admittance outer-tier conversion matrices. To obtain the impedance matrix of a given block, a small-signal test current will be sequentially introduced in parallel at the frequencies $m \omega_{o}+\Omega$, where $m=-M$ to $M$, at the open-circuited port. For instance, in the case of Fig. 7(a), the test current is introduced between the two terminals of the each open-circuited port, as shown in Fig. 8(a).

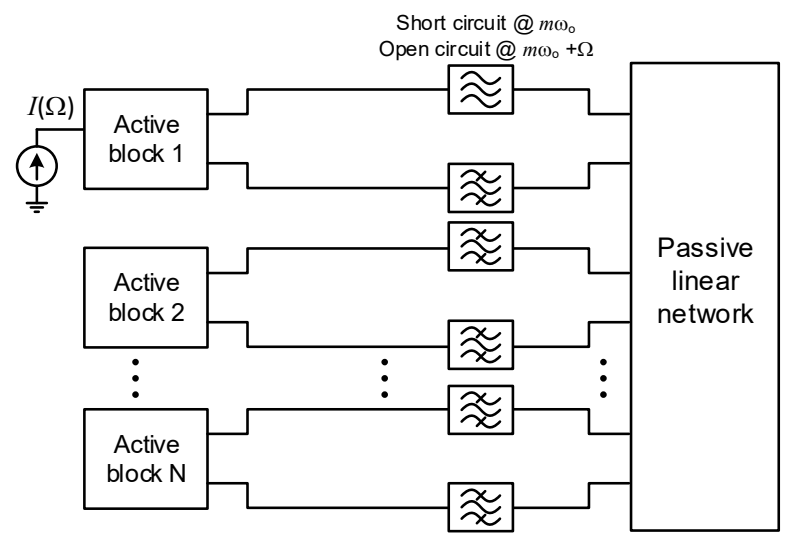

(a)

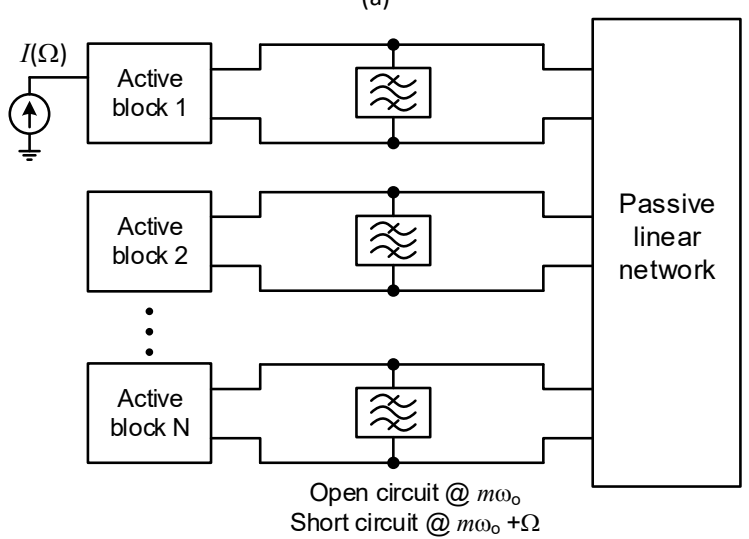

(b)

Fig. 7. Large-signal stability analysis under open-circuit and short-circuit terminations. These terminations should not affect the harmonic loads at $m \omega_{0}$, where $-M \leq m \leq M$. The diagram shows the test current used to obtain the closed-loop transfer functions. (a) Open-circuit case. (b) Short-circuit case.

For each $m$, one will perform a conversion-matrix analysis [53]-[54] to calculate the voltage drop at the frequencies $l \omega_{0}+\Omega$, where $l=-M$ to $M$. The element $Z_{A, n}(l, m)$ of the matrix is the ratio between voltage drop at $l \omega_{0}+\Omega$ and the current at $m \omega_{0}+\Omega$. When using commercial software, one should take into account that $I(-m, 1)=I^{*}(m,-1)$. The impedance-type outer-tier conversion matrix describing the block $n$ will be:

$$
\left[Z_{A, n}\right]=\left[\begin{array}{ccc}
Z_{A, n}(-M,-M) & \ldots & Z_{A, n}(-M, M) \\
\vdots & \vdots & \vdots \\
Z_{A, n}(M,-M) & \ldots & Z_{A, n}(M, M)
\end{array}\right]
$$

which relates the increments of the sideband voltages and currents as follows:

$$
\left[\begin{array}{c}
\Delta V(-M) \\
\vdots \\
\Delta V(M)
\end{array}\right]=\left[\begin{array}{ccc}
Z_{A, n}(-M,-M) & \ldots & Z_{A, n}(-M, M) \\
\vdots & \vdots & \vdots \\
Z_{A, n}(M,-M) & \ldots & Z_{A, n}(M, M)
\end{array}\right]\left[\begin{array}{c}
\Delta I(-M) \\
\vdots \\
\Delta I(M)
\end{array}\right]
$$

where $\Delta V(m)=\Delta V\left(m \omega_{o}+\Omega\right), \Delta I(m)=\Delta I\left(m \omega_{o}+\Omega\right)$. Then, the characteristic system is written as: 


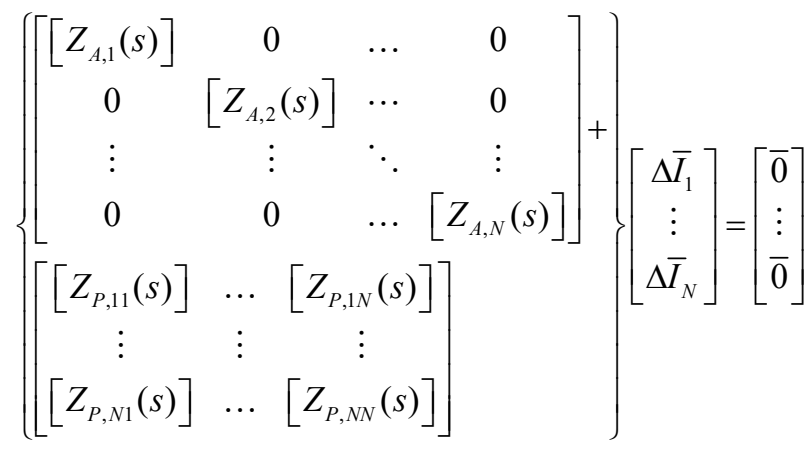

where $\left[Z_{P, n, s}\right]$ are the $M \times M$ submatrices composing the passive linear matrix $\left[Z_{P}(s)\right]$ and $\Delta \bar{I}_{n}$ is composed by the sideband currents at the $n^{\text {th }}$ block. They are given by:

$$
\begin{aligned}
{\left[Z_{P, n, s}\right] } & =\left[\begin{array}{cccc}
Z_{n, s}(-M,-M) & 0 & \ldots & 0 \\
0 & Z_{n, s}(-M+1,-M+1) & \ldots & 0 \\
\vdots & \vdots & \ddots & \vdots \\
0 & 0 & \ldots & Z_{n, s}(M, M)
\end{array}\right] \\
\Delta \bar{I}_{n} & =\left[\begin{array}{c}
\Delta I_{n}(-M) \\
\vdots \\
\Delta I_{n}(M)
\end{array}\right]
\end{aligned}
$$

Because the active blocks are stable under open-circuit terminations, none of the functions $Z_{A, n}(l, m)$ can have any poles in the RHS. Note that the elements $Z_{A, n}(l, m)$ actually correspond to transfer functions calculated within the same block and sharing the same denominator. Thus, the characteristic determinant associated with system (13) cannot exhibit any poles on the RHS. The characteristic system can be compactly written as:

$$
\left\{\left[Z_{A}(s)\right]+\left[Z_{P}(s)\right]\right\} \Delta \bar{I}=0
$$

The characteristic determinant is:

$$
\operatorname{det}\left\{\left[Z_{A}(s)\right]+\left[Z_{P}(s)\right]\right\}=0
$$

As in small-signal conditions, a normalization effect can be achieved multiplying system (15) by the inverse of the passive linear matrix $\left[Z_{P}(s)\right]$, which cannot introduce any RHS poles.

Now the case of a system composed by $N$ blocks that are SCstable will be considered. Each block $n$ will be described with an admittance-type conversion matrix. For this calculation, the block is connected to the system at the steady-state frequencies only, that is, at $m \omega_{o}$, where $m=-M$ to $M$. Then, a small-signal test voltage will be sequentially introduced in series at the frequencies $m \omega_{0}+\Omega$, where $m=-M$ to $M$ [Fig. 8(b)], at the branch that was previously short-circuited in Fig. 7(b). For each $m$, one will calculate the current through a voltage excitation at the frequencies $l \omega_{o}+\Omega$, where $l=-M$ to $M$. The element
$Y_{A, n}(l, m)$ of the matrix is the ratio between the current at $l \omega_{0}+\Omega$ and the voltage at $m \omega_{0}+\Omega$.

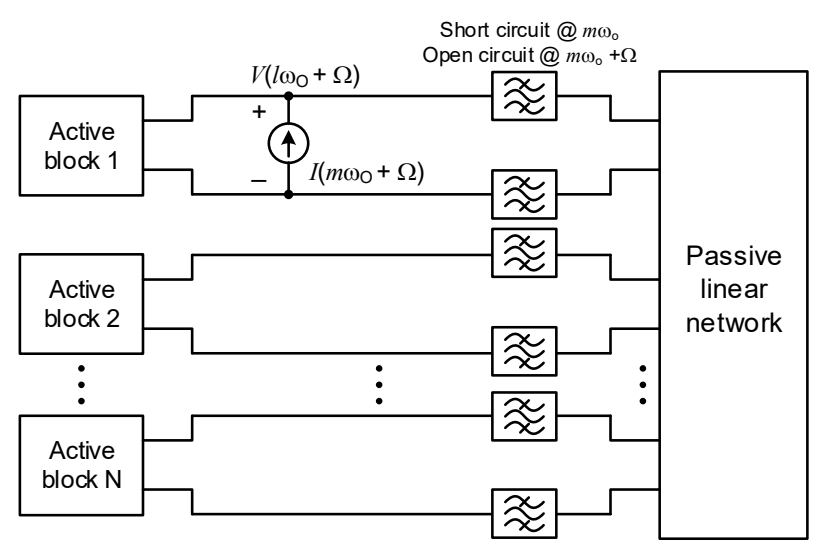

(a)

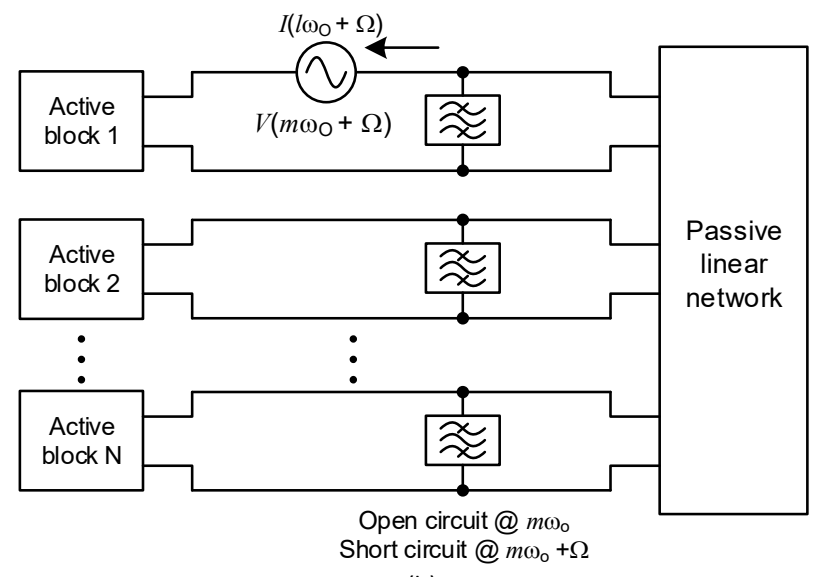

(b)

Fig. 8. Calculation of the outer-tier impedance/admittance conversion matrices of the individual blocks. Note that the blocks are connected at the periodic steady state only (frequencies $m \omega_{0}$, where $-M \leq m \leq M$ ). (a) OC-stable case. The element $Z_{A, n}(l, m)$ of the impedance matrix is the ratio between the voltage at $l \omega_{o}+\Omega$ and the current at $m \omega_{o}+\Omega$. (b) SC-stable case. The element $Y_{A, n}(l, m)$ of the admittance matrix is the ratio between the current at $l \omega_{o}+\Omega$ and the voltage at $m \omega_{0}+\Omega$.

When using commercial software, one should take into account that $V(-m, 1)=V^{*}(m,-1)$. Following a procedure analogous to (11)-(16), one obtains the characteristic system:

$$
\left\{\left[Y_{A}(s)\right]+\left[Y_{P}(s)\right]\right\} \Delta \bar{V}=0
$$

where $\Delta \bar{V}$ is the vector of sideband components of the node voltages. And the characteristic system is:

$$
\operatorname{det}\left\{\left[Y_{A}(s)\right]+\left[Y_{P}(s)\right]\right\}=0
$$

As stated, it is possible to consider two or more ports per active block, which will simply involve increasing the dimension of the matrices in (16) and (18).

\section{B. Practical implementation}

Section III.A demonstrated the absence of RHS poles in the characteristic determinant. In practice, one may directly 
calculate $\left[Z_{T}\right]=\left[Z_{A}\right]+\left[Z_{P}\right]$, in the case of OC-stable blocks, or $\left[Y_{T}\right]=\left[Y_{A}\right]+\left[Y_{P}\right]$, in the case of SC-stable blocks. This requires a template in commercial software and an in-house program to import the data and build-up the outer-tier impedance/admittance matrix.

Fig. 9 presents a template example to illustrate the implementation in ADS [55]. For briefness, only the case of OC-stable blocks is considered, since the extension to $\mathrm{SC}-$ stable blocks is straightforward. As shown in Fig. 9(a), the analysis ports (corresponding to those of the $\mathrm{OC}-$-stable blocks) are defined between the nodes Vtest_pn and ground, where $\mathrm{n}$ goes from 1 to $\mathrm{N}$. The circuit is analyzed with the conversionmatrix approach, or large-signal small-signal analysis. The test currents [Fig. 9(b)] used to calculate the full outer-tier impedance matrix are denoted Istab_pi k, where i, going from 1 to $\mathrm{N}$, refers to the injection node and $\mathrm{k}$ refers to the injection frequency. In Fig. 9 the injection node is 1, though an analogous analysis should be carried out injecting at the two other nodes. The test currents [Fig. 9(b)] are introduced sequentially at the mixing frequencies $(-\mathrm{M}, 1),(-\mathrm{M}+1,1), \ldots,(0,1),(\mathrm{M}, 1)$, where $M$ is the number of harmonic terms considered in the HB simulation. These frequencies are ranked in growing order. Thus, the index $\mathrm{k}$ in Istab pi $\mathrm{k}$, goes from 1 to $\mathrm{Q}=2 \mathrm{M}+1$. To keep the schematic in Fig. 9 at a reasonable size, the number of harmonic terms has been limited to $\mathrm{M}=3$ (only for this illustration), which provides a total of $Q=7$ test currents. These currents are activated sequentially by means of the sweep shown in Fig. 9(b). Depending on the value of aux_index, only one of the parameters Ipk, where $\mathrm{k}$ goes from 1 to $\overline{7}$, is different from zero, and activates the test current Istab_pn_k.

In the large-signal small-signal simulation, the small-signal frequency (associated with the index 1 of the mixing terms) should be swept from near DC to the fundamental frequency. To calculate the full outer-tier impedance matrix, for each Istab_pi_k one should obtain the voltage increment in each node $\mathrm{n}$ (going from 1 to $\mathrm{N}$ ) at each analysis frequency $\mathrm{q}$ (going from 1 to $\mathrm{Q})$. The excitation at port $\mathrm{i}$ and frequency $\mathrm{k}$ allows calculating the column $(\mathrm{i}-1) \mathrm{Q}+\mathrm{k}$ of the impedance matrix. The elements of this column are directly obtained by reading the voltage at each node $\mathrm{n}$ and frequency $\mathrm{q}$, and calculating the ratio:

$\mathrm{Z}[(\mathrm{n}-1) \mathrm{Q}+\mathrm{q},(\mathrm{i}-1) \mathrm{Q}+\mathrm{k}]=$ Vtest_pn(q)/Istab_pi_k (19)

Remember that the index q indicates the particular frequency in the ordered set of frequencies with mixing indexes $(-\mathrm{M}, 1),(-$ $\mathrm{M}+1,1), \ldots,(0,1), \ldots,(\mathrm{M}, 1)$. In practice, a common real value of Istab pi k is used to facilitate the calculation. A sequence of identical analyses is carried out injecting the test currents at the nodes $\mathrm{i}=1$ to $\mathrm{N}$. If a normalization is wished, the same procedure should be applied considering only the linear network that connects the OC-stable blocks. This will allow the calculation of $\left[Z_{P}\right]$.

The template is generated only once and reutilized for other systems. The Matlab script reads the tab-delimited ASCII files exported from ADS. The data read in Matlab must be rearranged due to the particular structure of the file produced by ADS when exporting the simulation results. The values of the swept parameters are placed in columns in the exported files and the impedance values (19) are interleaved in the form of successive vectors. At each value of the swept perturbation frequency $\Omega$, the vectors must be suitably arranged to build the impedance matrix.

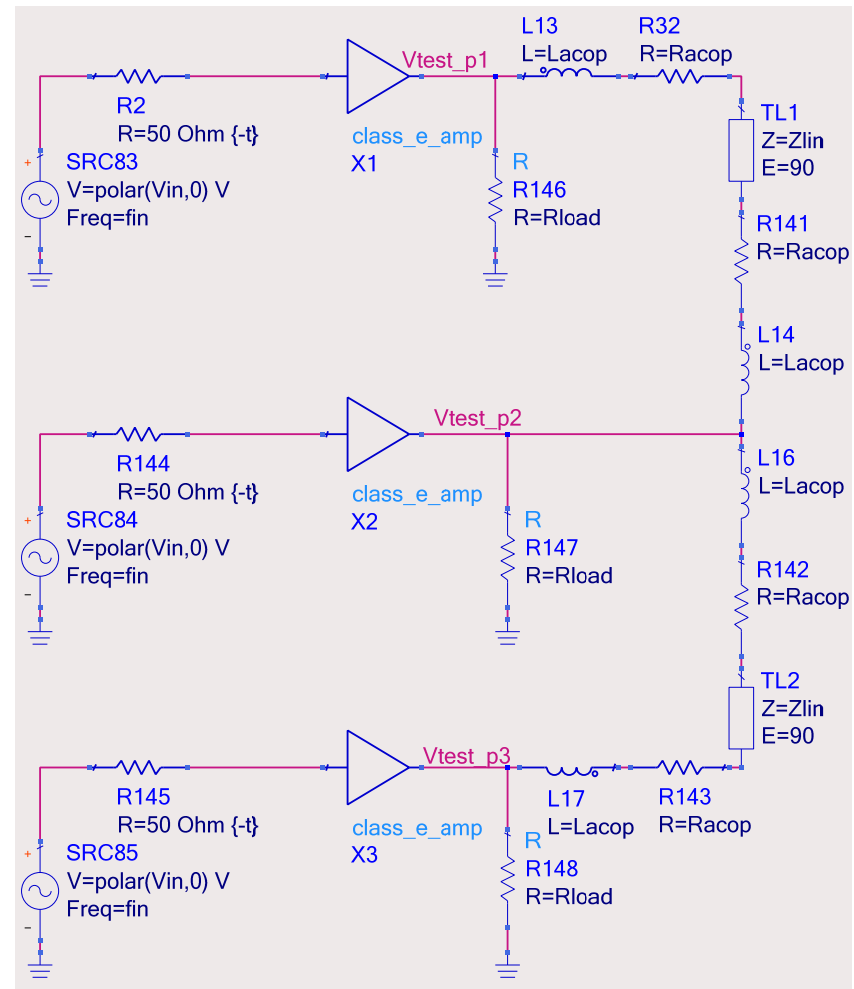

(a)

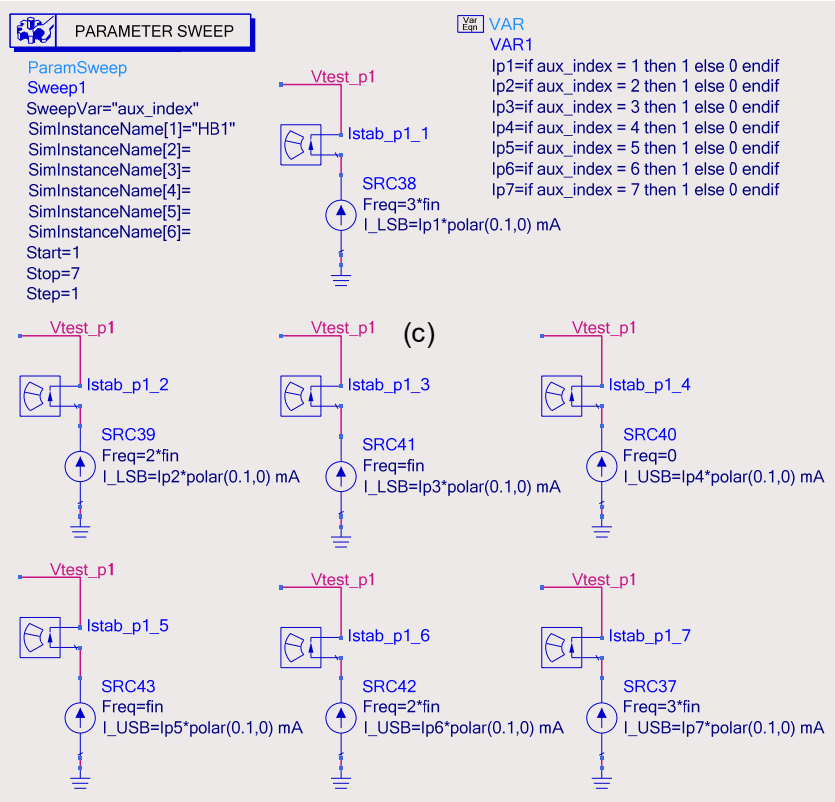

(b)

Fig. 9. Template example illustrating the calculation of the outer-tier impedance matrix. (a) The analysis ports (corresponding to those of the OC-stable blocks) are defined between the nodes Vtest pn and ground, where $\mathrm{n}$ goes from 1 to $\mathrm{N}$. (b) Sequential activation of the test currents by means of a sweep. Depending on the value of aux_index, only one of the parameters Ipk, where $\mathrm{k}$ goes from 1 to 7 , is different from zero, and activates the test current Istab pn k k.

\section{Application example}

For the large-signal stability analysis of the system in Fig. 1(a), the feedback network that had been added to the third 
amplifier is removed. Thus, the three power amplifiers are stable for all the $V_{G S}$ values. The analysis will be carried out versus the input voltage $V_{i n}$ at the frequency $f_{i n}=800 \mathrm{MHz}$ and gate-bias voltage $V_{G S}=0.12 \mathrm{~V}$. Initially, the coupling resistor is $R=5 \Omega$. Taking into account that the three power amplifiers are stable in standalone operation, the OC stability of the circuit blocks was initially evaluated and, as shown in the following, this OC-stability test was successful.

The OC stability of the three identical amplifiers (taken as active blocks) is analyzed by disconnecting them from the coupling network at the sideband frequencies $m \omega_{0}+\Omega$ (Fig. 10), though they are kept connected at the frequencies $m \omega_{o}$ of the periodic steady state. Although the three amplifiers are identical, they are not in the same operation conditions: the outermost power amplifiers are coupled to the middle amplifier only, whereas the middle amplifier is coupled to the two outermost amplifiers.
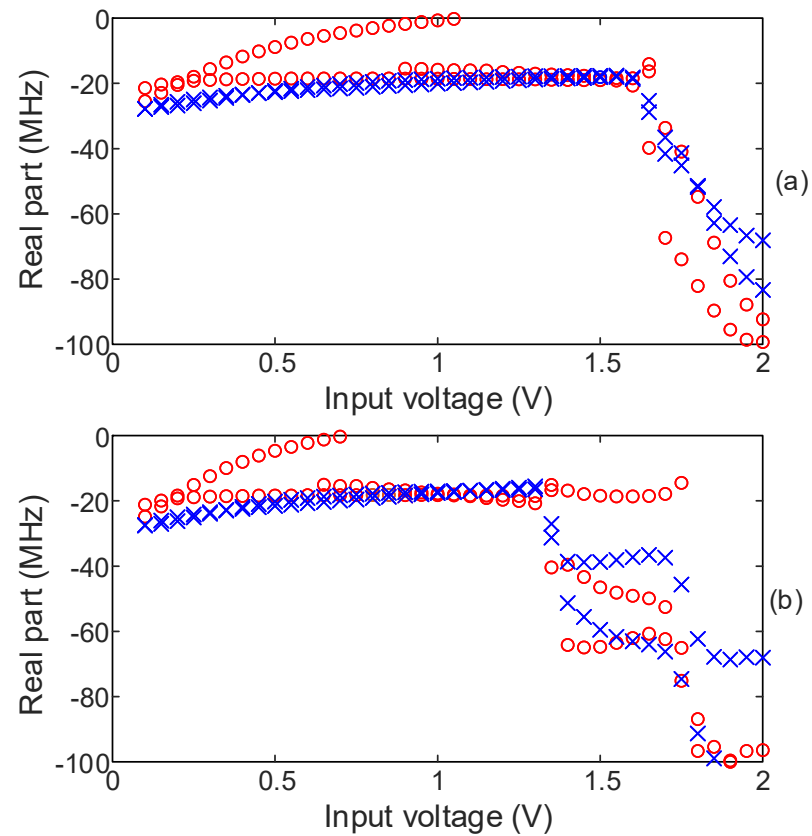

Fig. 10. Large-signal analysis. Verification of the open-circuit stability of the power amplifiers in Fig. 2(a) through conventional pole-zero identification, applied to a transfer function. The stability information is in the poles $(\times)$. The open-circuit condition is imposed at the sidebands only; the amplifiers are coupled at the periodic steady state obtained for each $V_{i n}$. (a) Real part of the dominant poles and zeroes (o) versus $V_{i n}$ for the first amplifier (in equivalent operation conditions to the third). (b) Real part of the dominant poles and zeroes versus $V_{i n}$ for the middle amplifier.

Under open-circuit terminations, the three amplifiers are stable for all the $V_{\text {in }}$ values, as verified with pole-zero identification. This identification is applied in a conventional manner to a transfer function calculated by connecting a smallsignal test current at the frequency $\Omega$ (incommensurable with $\omega_{i n}=2 \pi f_{i n}$ ) in parallel at the transistor gate terminal. Fig. 10(a) presents the variation of the real part of the dominant poles of the first and third amplifier (having equivalent operation points) versus $V_{i n}$. Identical results are obtained, so only one of the analyses in presented. Fig. 10(b) presents the variation of the real part of the dominant poles in the middle amplifier versus $V_{i n}$. The three amplifiers are stable in open-circuit conditions for all the $V_{i n}$ values. The same conclusion is obtained when the test current is introduced at other device terminals. Thus, the stability of the entire system can be analyzed using the impedance-based determinant (16).

Fig. 11 presents the Nyquist plots obtained for two different $V_{i n}$ values before and after the first Hopf bifurcation that gives rise to instability. For $V_{i n}=1.07 \mathrm{~V}$ [Fig. 11(a)] the Nyquist plot does not encircle the origin, so the system is stable. In contrast, for $V_{\text {in }}=1.075 \mathrm{~V}$ [Fig. 11(b)], the Nyquist plot encircles the origin in clockwise sense, so the system is unstable. Fig. 12 presents the identification of the characteristic determinant (16) for the same two $V_{i n}$ values. Thus, as in the case of the smallsignal stability analysis, it is possible to use both the Nyquist criterion, which has the advantage of requiring a single frequency sweep, and pole-zero identification.

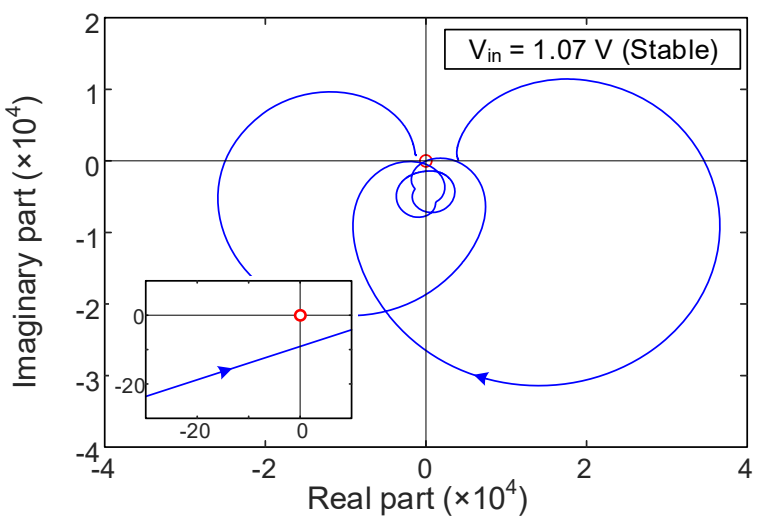

(a)

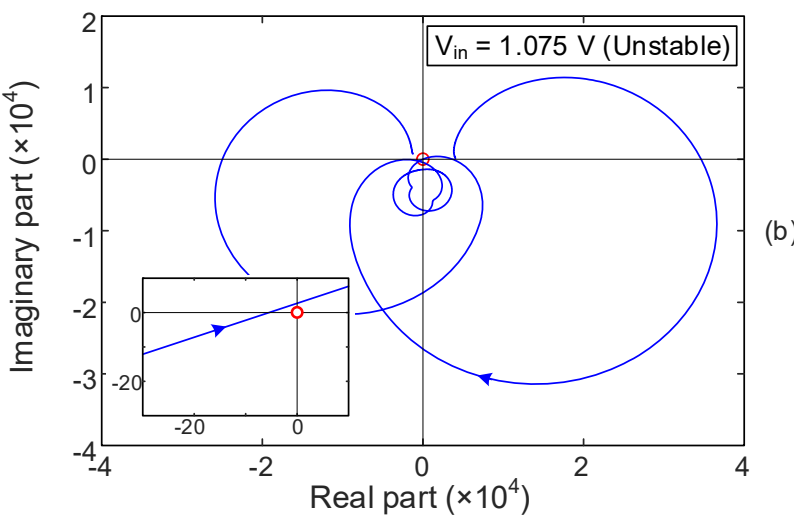

Fig. 11 Large-signal stability analysis of the circuit in Fig. 2(a), without seriesfeedback in the third amplifier, through the characteristic determinant in (16). (a) Nyquist plot for the input voltage $V_{i n}=1.07 \mathrm{~V}$ (stable operation). Expanded view in the inset. (b) Nyquist plot for the input voltage $V_{\text {in }}=1.075 \mathrm{~V}$ (unstable operation).

Fig. 13(a) presents the variation of the dominant zeroes and poles of the characteristic determinant (16) versus $V_{i n}$. As can be seen, the coupling effects give rise to two secondary Hopf bifurcations [12], [15]-[16], occurring at the $V_{\text {in }}$ values $1.075 \mathrm{~V}$ and $1.145 \mathrm{~V}$. At the first bifurcation $(1.075 \mathrm{~V})$, a pair of complex conjugate zeroes at $561 \mathrm{MHz}$ crosses the imaginary axis to the RHS. At the second bifurcation $(1.145 \mathrm{~V})$, a second pair of complex conjugate zeroes at $237 \mathrm{MHz}$ crosses the imaginary axis to the RHS. As stated, although the power amplifiers are identical, they are not in the same operation conditions and the 
two Hopf bifurcations take place at different $V_{\text {in }}$ values. For better insight, Fig. 13(b) presents the variation of the real part of the dominant zeroes of (16) versus $V_{i n}$.

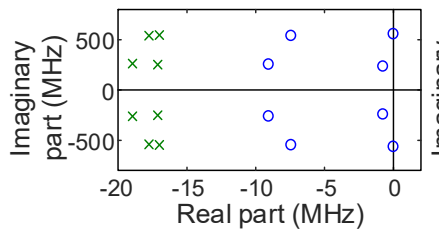

(a)

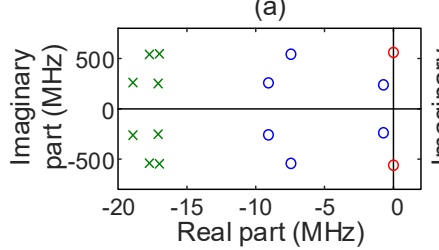

(c)

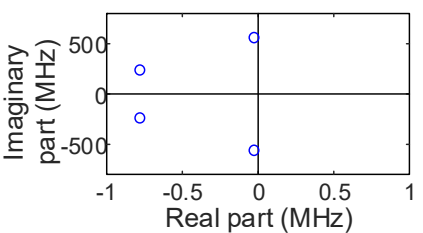

(b)

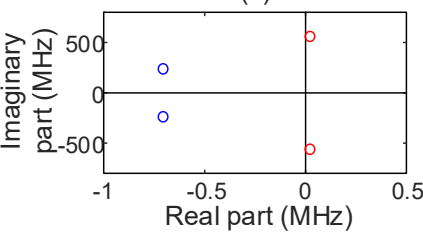

(d)
Fig. 12. Large-signal stability analysis. Pole-zero identification of the characteristic determinant for the two cases considered in Fig. 10. The stability information is in the zeroes. (a) Poles $(\times)$ and zeroes (o) for $V_{\text {in }}=1.07 \mathrm{~V}$ (stable operation). (b) Expanded view about the dominant zeroes. (c) Input voltage $V_{\text {in }}=1.075 \mathrm{~V}$ (unstable operation). (d) Expanded view about the dominant zeroes.

The above result has been validated through pole-zero identification, applied in a conventional manner to a transfer function calculated at the gate terminal of the transistor in the middle amplifier. Fig. 13(c) presents the variation of the real part of the dominant poles versus $V_{i n}$. Even though the identified functions are very different, there is a full agreement between the poles obtained through conventional pole-zero identification (applied to a transfer function) and the zeroes of the characteristic determinant (16).

When increasing the coupling resistor, or equivalently, decreasing the coupling effects, the standard pole-zero identification faces a problem of limited observability. To show this, the coupling resistor between the second and third amplifier will be increased to $R=330 \Omega$ in the next experiment.

Fig. 14 presents the stability analysis of the whole system when identifying the characteristic determinant [in (a)] and when applying conventional pole-zero identification at two different nodes of the third amplifier [in (b) and (c)]. These nodes are the gate terminal, in Fig. 14(b), and the node at which the third amplifier is connected to the coupling network, in Fig. 14(c). The zeroes of the determinant, shown in Fig. 14(a), and the poles in Fig. 14(b) are identical. However, the latter are quasi-cancelled with RHS zeroes due to the limited observability. The situation is more severe in the Fig. 14(c), where the unstable poles have completely disappeared and the instability cannot be detected.
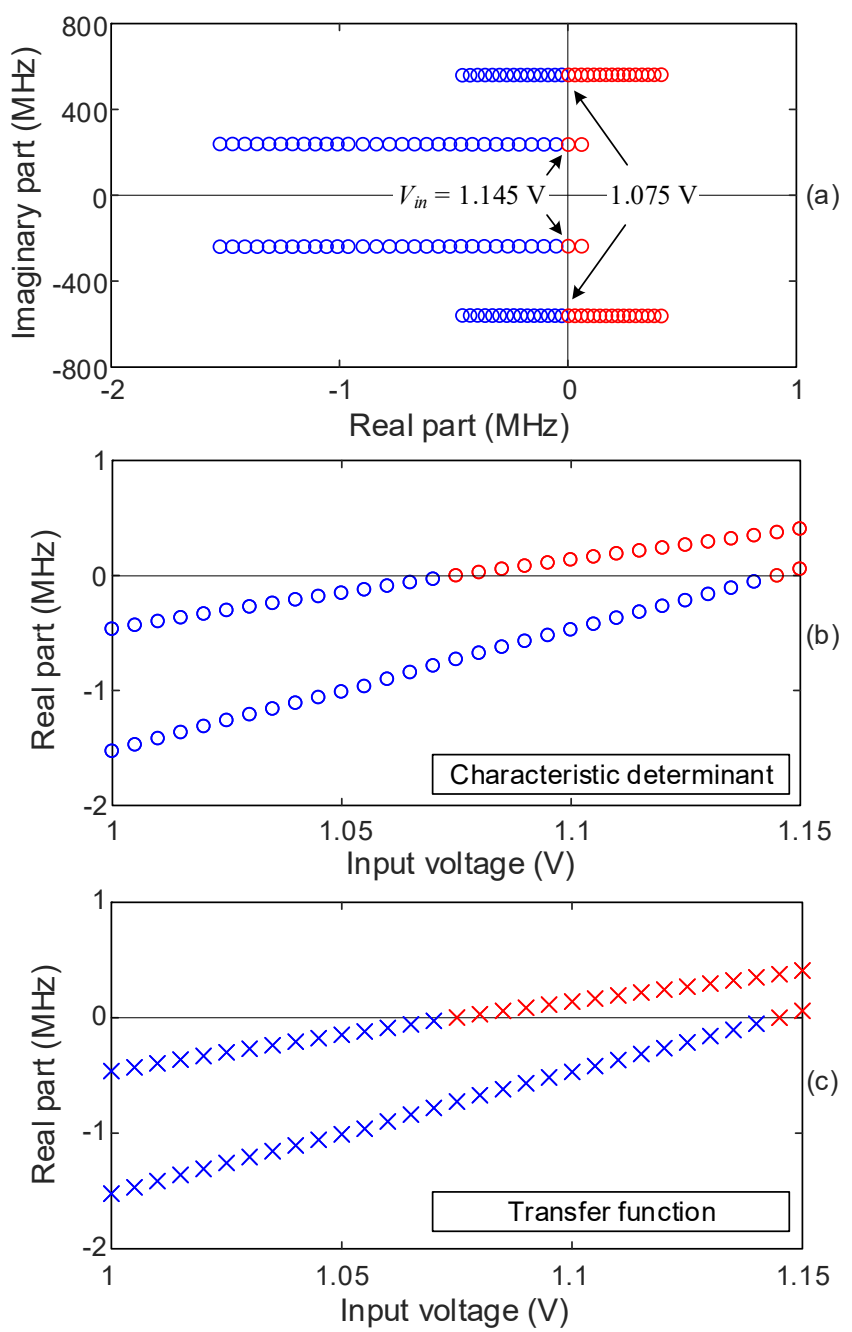

Fig. 13. Large-signal stability analysis. (a) Variation of the dominant zeroes (o) and poles $(\times)$ of the characteristic determinant (16) versus $V_{i n}$. The stability information is in the zeroes. (b) Variation of the real part of the dominant zeroes of (16) versus $V_{i n}$. (c) Variation of the real part of the dominant poles obtained through conventional pole-zero identification applied to a transfer function. This is obtained by introducing the test current at the gate terminal of the third amplifier. The stability information is in the poles.

One of the advantages of the new method is that, by construction, it prevents the coexistence of RHS zeroes and RHS poles and, thus, the zero-pole cancellations or quasicancellations that are the main limitation of the powerful polezero identification [26]-[31]. Here the conventional pole-zero identification is applied to verify the OC or SC stability of the composing blocks, which can be done reliably due to the limited block size and complexity. Then, the full system stability is analyzed at once using the characteristic determinant. With conventional pole-zero identification, the analysis of the whole structure would be carried out through several evaluations at different locations, which may be subject to problems of limited observability. 

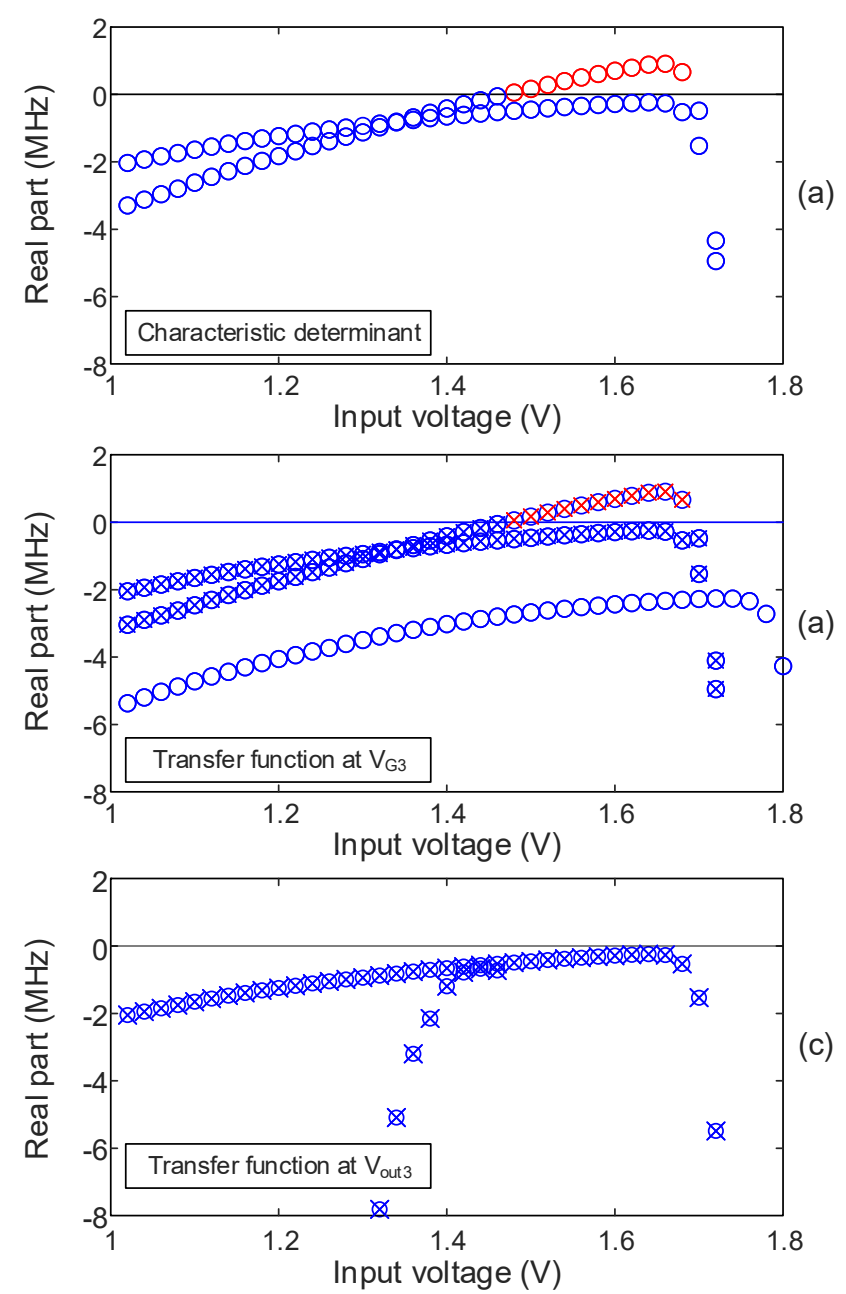

Fig. 14. Large-signal stability analysis under weak coupling effects between the second and third amplifier $(R=330 \Omega)$. (a) Analysis versus $V_{i n}$ through the identification of the characteristic determinant (16). The stability information is in the zeroes (o). (b) Pole-zero identification when the test current is connected in parallel at the gate terminal of the third amplifier. The stability information is in the poles $(\times)$. (c) Pole-zero identification when the test current is introduced at the node where the third amplifier is connected to the coupling network. The unstable poles have completely disappeared, so the instability cannot be detected.

Fig. 15 presents a photograph of the practical implementation of the coupled system. It was manufactured on RO4003C substrate $\left(\varepsilon_{\mathrm{r}}=3.5, \mathrm{~h}=32 \mathrm{mil}\right)$, with Avago $\AA$ ATF50189 FETs, air core inductors by Coilcraft $\AA$ and ATC $®$ ceramic capacitors. All the amplifiers and coupling networks are identical, with $R=5 \Omega$. As stated, when measured in standalone operation, the amplifier exhibits $24 \mathrm{dBm}$ output power and $60 \%$ efficiency. However, the performance is strongly affected by the coupling network. Fig. 16(a) presents the measured spectrum at the input frequency $800 \mathrm{MHz}$ and amplitude $V_{i n}=1 \mathrm{~V}$, which is compared with the one obtained in HB simulations. In agreement with the predictions of the stability analysis of Fig. 13, the spectrum corresponds to a (stable) periodic solution, with lines at multiples of the input frequency $800 \mathrm{MHz}$. There is a good correspondence between the simulated and measured results, which indicates that the models are reliable. For the rest of measurements, a $10 \mathrm{~dB}$ coaxial directional coupler is used, which ensures the same loading conditions in the three amplifiers.

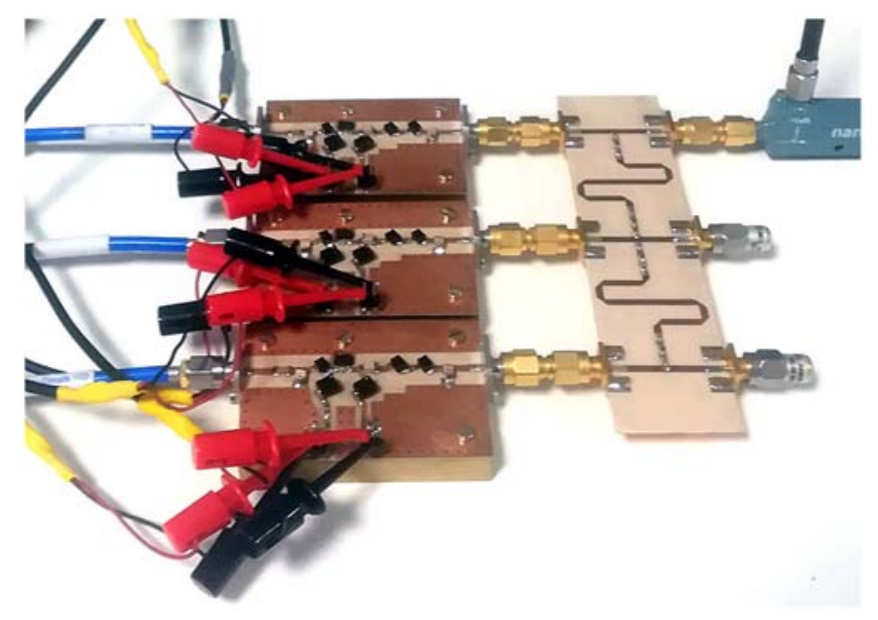

Fig. 15. Practical implementation of the coupled system with identical amplifiers and coupling networks. They were manufactured on RO4003C substrate $\left(\varepsilon_{\mathrm{r}}=3.5, \mathrm{~h}=32 \mathrm{mil}\right)$, with Avago ${ }$ ATF50189 FETs, air core inductors by Coilcraft ${ }^{\circledR}$ and $A T C \circledR$ ceramic capacitors. The output spectrum was measured through a NARDA $4011 \mathrm{C}-10$ coaxial directional coupler.

When the periodic solution (at the input frequency) obtained with default HB is unstable, this solution coexists with a different (stable) steady-state solution. The stable solution is the one observed in the measurements. Thus, the qualitative change in the measured solution defines the stability limit of the periodic solution obtained with HB. In the case of the system considered here, when increasing $V_{i n}$, the periodic solution is measured up to $V_{\text {in }}=1.1 \mathrm{~V}$. From this voltage value, the measured solution is not periodic, but quasi-periodic [Fig. 16(b) and Fig. 16(c)], due to the mixing of the input signal with two self-generated oscillations. Thus, the measured stability limit is $V_{i n}=1.1$ V. From Fig. 13, the simulated stability limit, at which the first pair of complex-conjugate zeroes cross to the RHS, is $V_{\text {in }}=1.075 \mathrm{~V}$, so there is a good correspondence.

In general, one can gather the type of steady-state solution that will be obtained in the measurements from the roots of the characteristic determinant of the (default) periodic solution. In the stability analysis of Fig. 13(a), a pair of complex-conjugate zeroes at $561 \mathrm{MHz}$ cross to the RHS at $V_{\text {in }}=1.07 \mathrm{~V}$, and a second pair of complex-conjugate zeroes at $237 \mathrm{MHZ}$ cross to the RHS at $V_{i n}=1.145 \mathrm{~V}$. In the spectrum of Fig. 16(b), obtained for $V_{i n}=1.12 \mathrm{~V}$, there are two concurrent oscillation frequencies, which is consistent with the two pairs of complexconjugates zeroes crossing to the RHS in Fig. 13(a). The frequencies of these two pairs of zeroes, $561 \mathrm{MHz}$ and $237 \mathrm{MHz}$, are close to a 2:1 relationship. Although the oscillation generated at $V_{i n}=1.145 \mathrm{~V}$ in Fig. 13(a) will be initially unstable, after the occurrence of two secondary Hopf bifurcations, concurrent stable oscillations are likely to be observed experimentally, as described in [56]-[57]. In the measurement of Fig. 16(b), the two oscillations are locked, as gathered from the frequency values shown in the spectrum. Fig. $16(\mathrm{c})$ presents the spectrum obtained for $V_{i n}=1.26 \mathrm{~V}$, which also corresponds to a quasiperiodic solution, in agreement with Fig. 13. Thus, there is a very good consistency between 
simulations and measurements. Nevertheless, the most reliable validation is the one performed versus an independent analysis method, such as pole-zero identification, as done through the entire manuscript. This is the only way to ensure that there are no discrepancies in the input elements and parameters resulting from inaccuracies in the models of the active and passive elements.

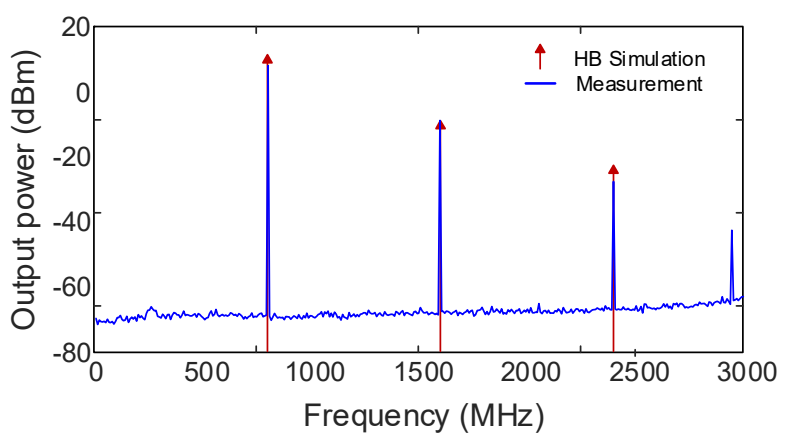

(a)

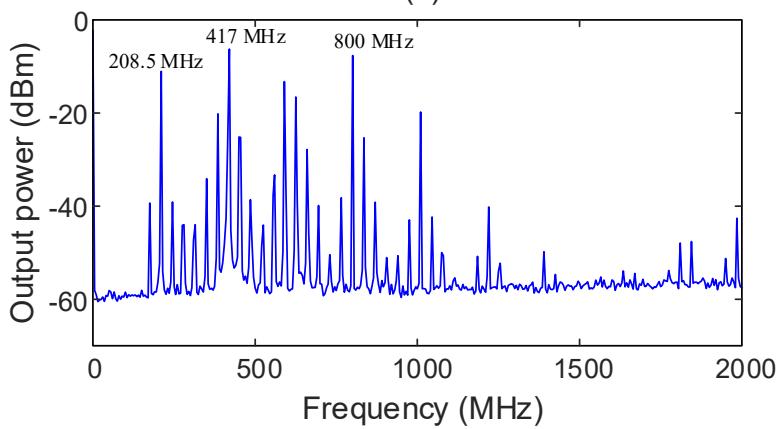

(b)

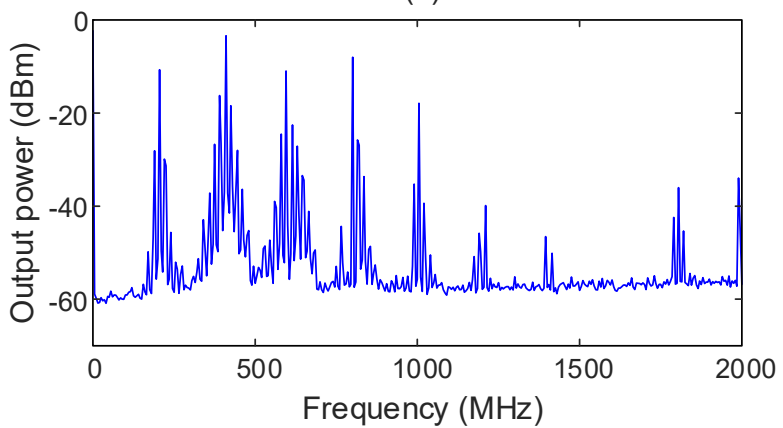

(c)

Fig. 16. Experimental measurements. (a) Spectrum for the input voltage $V_{\text {in }}=1.1 \mathrm{~V}$, measured without the $10 \mathrm{~dB}$ coaxial directional coupler (stable behavior). It is compared with the one obtained in HB. (b) Spectrum for $V_{\text {in }}=1.12 \mathrm{~V}$ (unstable behavior, with two locked self-generated oscillations). (c) Spectrum for $V_{i n}=1.26 \mathrm{~V}$ (unstable behavior).

\section{CONCLUSIONS}

A method has been presented for the small- and large-signal stability analysis of complex systems, composed by multiple active blocks. The method is based on a definition of the individual blocks that ensures that these blocks are stable under either open-circuit terminations or short-circuit terminations. This is verified with pole-zero identification, considering one or more ports per block. The total set of block ports constitute the $N$ reference ports considered in the calculation of the system characteristic determinant. The stability properties are defined by the zeroes of this determinant, which, by construction, cannot exhibit any poles in the right-hand side of the complex plane. The extension to large-signal regime requires the calculation of an outer-tier conversion matrix per active block. The stability properties can be obtained by applying the Nyquist criterion or pole-zero identification to the characteristic determinant of the full system. The procedure has been successfully validated with independent simulations and with measurements. It can be implemented on commercial HB and provides a determinant function that does not suffer from any uncertainties associated with the possible cancellations or quasi-cancellations of RHS zeroes and poles.

\section{REFERENCES}

[1] S. Jeon, A. Suárez, R. Rutledge, "Global stability analysis and stabilization of a Class-E/F amplifier with a distributed active transformer," IEEE Trans. Microw. Theory Techn., vol. 53, no. 12, Dec. 2005, pp. 3712-3722.

[2] R. Quéré, E. Ngoya, M. Camiade, A. Suarez, M. Hessane and J. Obregon, "Large signal design of broadband monolithic microwave frequency dividers and phase-locked oscillators," IEEE Trans. Microw. Theory Techn., vol. 41, no. 11, pp. 1928-1938, Nov., 1993

[3] S. Mons, J.-C. Nallatamby, R. Queré, P. Savary, and J. Obregon, "A unified approach for the linear and nonlinear stability analysis of microwave circuits using commercially available tools," IEEE Trans. Microw. Theory Techn., vol. 47, no. 12, pp. 2403-2409, Dec. 1999.

[4] Sanggeun Jeon, A. Suarez and D. B. Rutledge, "Analysis and elimination of hysteresis and noisy precursors in power amplifiers," IEEE Trans. Microw. Theory Techn., vol. 54, no. 3, pp. 1096-1106, March 2006.

[5] J. de Cos, A. Suárez, J.A. García, "Hysteresis and Oscillation in HighEfficiency Power Amplifiers," IEEE Trans. Microw. Theory Techn., vol. 63, no. 12, pp. 4284-4296, Dec., 2015.

[6] L. Pantoli, G. Leuzzi, A. Santarelli and F. Filicori, "Stability Analysis and Design Criteria of Paralleled-Device Power Amplifiers Under LargeSignal Regime," IEEE Trans. Microw. Theory Techn., vol. 64, no. 5, pp. 1442-1455, May 2016.

[7] S. Colangeli, W. Ciccognani and E. Limiti, "Algorithmic Test of the Unconditional Stability of Three-Port Networks," IEEE Trans. Microw. Theory Techn., vol. 66, no. 12, pp. 5197-5205, Dec. 2018.

[8] S. Colangeli, R. Giofrè, W. Ciccognani and E. Limiti, "A multi-finger modeling approach to correctly predict the inherent stability of a custom active device," IEEE MTT-S Int. Microwave Symp., Honololu, HI, 2017, pp. 1784-1786.

[9] A. Suarez, "Check the Stability: Stability Analysis Methods for Microwave Circuits," IEEE Microw. Magazine, vol. 16, no. 5, pp. 69-90, June 2015.

[10] F. Bonani and M. Gilli, "Analysis of stability and bifurcations of limit cycles in Chua's circuit through the harmonic-balance approach," IEEE Trans. Circuits and Systems-I, vol. 46, no. 8, pp. 881-890, Aug., 1999.

[11] S. ver Hoeye, L. Zurdo and A. Suarez, "New nonlinear design tools for self-oscillating mixers," IEEE Microw. and Wireless Components Letters, vol. 11, no. 8, pp. 337-339, Aug. 2001.

[12] J. M. T. Thompson, H. B. Stewart, Nonlinear Dynamics and Chaos, John Wiley \& Sons, Feb 15, 2002.

[13] Z. Heshmati, I. C. Hunter and R. D. Pollard, "Microwave Parametric Frequency Dividers With Conversion Gain," IEEE Trans. Microw. Theory Techn., vol. 55, no. 10, pp. 2059-2064, Oct. 2007.

[14] A. Suárez, R. Quéré, Stability Analysis of Nonlinear Microwave Circuits, Artech House, Norwood (Ma), Jan. 2003.

[15] A. Suárez, Analysis and Design of Autonomous Microwave Circuits, IEEE-Wiley, Hoboken, (NJ) Jan. 2009.

[16] V. Rizzoli and A. Neri, "State of the art and present trends in nonlinear microwave CAD techniques," IEEE Trans. Microw. Theory Techn., vol. 36, no. 2, pp. 343-356, Feb., 1988.

[17] K. S. Kundert, "Introduction to RF simulation and its application," IEEE J. Solid State Circuits, vol. 34, no. 9, pp. 1298-1319, Sep., 1999. 
[18] Guillermo González, Microwave Transistor Amplifiers: Analysis and Design, $2^{\text {nd }}$ Edition, Prentice Hall , Upper Saddle River, New Jersey, USA, 1996.

[19] D. Woods, "Reappraisal of the unconditional stability criteria for active 2port networks in terms of S parameters," IEEE Trans. on Circuits and Systems, vol.23, no. 2, pp. 73-81, Feb., 1976.

[20] A. Platzker, W. Struble and K. T. Hetzler, "Instabilities diagnosis and the role of $\mathrm{K}$ in microwave circuits," IEEE MTT-S Int. Microwave Symp., Atlanta, GA, USA, 1993, pp. 1185-1188 vol.3.

[21] A. Platzker, W. Struble, "Rigorous determination of the stability of linear n-node circuits from network determinants and the appropriate role of the stability factor $\mathrm{K}$ of their reduced two-ports," Third International Workshop on Integrated Nonlinear Microwave and Millimeterwave Circuits, pp. 93-107, 5-7, Oct. 1994.

[22] W. Struble, A. Platzker, "A rigorous yet simple method for determining stability of linear N-port networks [and MMIC application]," 15th Gallium Arsenide Integrated Circuit (GaAs IC) Symposium, pp. 251 - 254, 1993.

[23] J. Rollett, "Stability and Power-Gain Invariants of Linear Twoports," IRE Trans. Circuit Theory, vol. 9, no. 1, pp. 29-32, Mar., 1962.

[24] K. Ogata, Modern Control Engineering, Englewood Cliffs, NJ, PrenticeHall, 1980.

[25] V. Rizzoli, A. Lipparini, "General stability analysis of periodic steadystate regimes in nonlinear microwave circuits," IEEE Trans. Microw. Theory Techn., vol. 33, no. 1, pp. 30 - 37, Jan. 1985.

[26] J. Jugo, J. Portilla, A. Anakabe, A. Suárez, and J. M. Collantes, "Closedloop stability analysis of microwave amplifiers," IEE Electronics Letters, vol. 37, no. 4, pp. 226-228, Feb. 2001.

[27] A. Anakabe, J. M. Collantes, J. Portilla, et al. "Analysis and elimination of parametric oscillations in monolithic power amplifiers," IEEE MTT-S Int. Microwave Symp. Seattle, WA, Jun. 2002, pp. 2181-2184.

[28] N. Ayllon, J. M. Collantes, A. Anakabe, I. Lizarraga, S. Soubercaze-Pun, S. Forestier, "Systematic approach to the stabilization of multitransistor circuits", IEEE Trans. Microw. Theory Techn., vol. 59, no. 8, pp. 20732082, Aug. 2011.

[29] J. M. Collantes, I. Lizarraga, A. Anakabe, J. Jugo, "Stability verification of microwave circuits through Floquet multiplier analysis," 2004 IEEE APCCAS, Taiwan, 2004, pp. 997-1000.

[30] J.M. Collantes, L. Mori, A. Anakabe, N. Otegi, I. Lizarraga, N. Ayllón, F. Ramírez, V. Armengaud, G. Soubercaze-Pun, "Pole-zero Identification: Unveiling the Critical Dynamics of Microwave Circuits Beyond Stability Analysis," IEEE Microw. Mag., vol. 20, no. 7, pp. 36-54, Jul., 2019.

[31] N. Ayllon, A. Anakabe, J. M. Collantes, G. Soubercaze-Pun, S. Forestier, "Sensitivity Enhancement in Pole-zero Identification Based Stability Analysis of Microwave Circuits," Workshop on Integrated Nonlinear Microwave and Millimetre-Wave Circuits, Malaga, 2008, pp. 75-78.

[32] A. Cooman, F. Seyfert, M. Olivi, S. Chevillard, L. Baratchart, "ModelFree Closed-Loop Stability Analysis: A Linear Functional Approach," IEEE Trans. Microw. Theory Techn., vol. 66, no. 1, pp. 73-80, Jan., 2018.

[33] C. Barquinero, A. Suárez, A. Herrera, J. L. García, "Complete stability analysis of multifunction MMIC circuits," IEEE Trans. Microw. Theory Techn., vol. 55, no. 10, pp. 2024-2033, Oct., 2007.

[34] A. Suárez, F. Ramírez, "Stability and Bifurcation Analysis of MultiElement Non-Foster Networks," IEEE Trans. Microw. Theory Techn., vol. 66, no. 4, pp. 1817-1830, Apr., 2018.

[35] A. Suárez, F. Ramírez, "Circuit-level Stability and Bifurcation Analysis of Non-Foster Circuits," IEEE MTT-S Int. Microwave Symp., Honolulu, HI, USA, June, 2017.

[36] M. M. Jacob and D. F. Sievenpiper, "Non-Foster Matched Antennas for High-Power Applications," in IEEE Transactions on Antennas and Propagation, vol. 65, no. 9, pp. 4461-4469, Sept. 2017.

[37] H. Mirzaei and G. V. Eleftheriades, "Unilateral non-Foster elements using loss-compensated negative-group-delay networks for guided-wave applications," 2013 IEEE MTT-S International Microwave Symposium Digest (MTT), Seattle, WA, 2013, pp. 1-4.

[38] Vasudev K. Aatre, Network theory and filter design, John Wiley \& Sons, 1981.

[39] D. Youla, Theory and Synthesis of Linear Passive Time-Invariant Networks, Cambridge University Press, 2015, Cambridge, UK.

[40] T. Deliyannis, Yichuang Sun, J.K. Fidler, Continuous-Time Active Filter Design, CRC Press, Boca Raton, USA, 2019.

[41] L.O. Chua, C.A. Desoer, E. S. Kuh, Linear and Nonlinear Circuits, McGraw-Hill, New York, 1987.

[42] G. Martinelli, "Realisation of a short-circuit-stable negative-resistance circuit with no internal bias supplies," Electronics Letters, vol. 2, no. 8, pp. 308-309, August 1966.
[43] M. Bakshi, V. Sule, M. Baghini, "Systems Theory Approach to Stabilization of Multiport Networks," Journal of Control and Systems Engineering, vol. 15, no. 1, pp 48-63, 2017.

[44] A. Antoniou, "Floating negative-impedance converters," IEEE Trans. Circuit Theory, vol. 19, no. 2, pp. 209-212, March 1972.

[45] A. Davies, "Stability Properties of a Negative Immittance Converter," IEEE Trans. Circuit Theory, vol. 15, no. 1, pp. 80-81, March 1968.

[46] J. Imbornone, M. Murphy, R. S. Donahue and E. Heaney, "New insight into subharmonic oscillation mode of GaAs power amplifiers under severe output mismatch condition," GaAs IC Symposium IEEE Gallium Arsenide Integrated Circuit Symposium. Orlando, FL, USA, 1996, pp. 307-310

[47] J. F. Imbornone, M. Murphy, R. S. Donahue, and E. Heaney, "New insight into subharmonic oscillation mode of GaAs power amplifiers under severe output mismatch condition," IEEE J. Solid-State Circuits, vol. 32, no. 9, pp. 1319-1325, Sep. 1997

[48] S. Dellier, R. Gourseyrol, J. Collantes, A. Anakabe, G. SoubercazePun, and K. Narendra, "Stability analysis of microwave circuits," IEEE Wireless Microw. Techn. Conf., Cocoa Beach, FL, USA, 2012, pp. 1-5.

[49] D. Qiao, D. Cho, Y. Zhao, T. Hung, D. Kimball, M. Li, and P. Asbeck, "Antenna impedance mismatch measurement and correction for adaptive CDMA transceivers," IEEE MTT-S Int. Microwave Symp., Long Beach, CA, USA, 2005, pp. 783-786.

[50] K. Hausmair, P. N. Landin, U. Gustavsson, C. Fager and T. Eriksson, "Digital Predistortion for Multi-Antenna Transmitters Affected by Antenna Crosstalk," IEEE Trans. Microw. Theory Techn., vol. 66, no. 3, pp. 1524-1535, Mar., 2018

[51] S. K. Dhar, A. Abdelhafiz, M. Aziz, M. Helaoui and F. M. Ghannouchi, "A Reflection-Aware Unified Modeling and Linearization Approach for Power Amplifier Under Mismatch and Mutual Coupling," IEEE Trans. Microw. Theory Techn., vol. 66, no. 9, pp. 4147-4157, Sept., 2018.

[52] J. Guckenheimer and P. Holmes, Nonlinear Oscillations, Dynamical Systems and Bifurcations of Vector Fields. New York: Springer-Verlag, 1990.

[53] V. Rizzoli, F. Mastri and D. Masotti, "General noise analysis of nonlinear microwave circuits by the piecewise harmonic balance technique," IEEE Trans. Microw. Theory Techn., vol. 42, no. 5, pp. 807-819, May, 1994.

[54] J. M. Paillot, J. C. Nallatamby, M. Hessane, R. Quéré, M. Prigent and J. Rousset, "A general program for steady state, stability, and FM noise analysis of microwave oscillators," IEEE MTT-S Int. Microwave Symp., 1990, pp. 1287-1290.

[55] Keysight Technologies, Advanced Design System (2020 U2), Santa Rosa, CA.

[56] J. de Cos, A. Suarez and F. Ramirez, "Analysis of Oscillation Modes in Free-Running Ring Oscillators," IEEE Trans. Microw. Theory Techn., vol. 60, no. 10, pp. 3137-3150, Oct. 2012.

[57] F. Ramírez, S. Sancho, A. Suárez, "Oscillation Modes in Free-Running Oscillators Loaded with Multi-Resonant Networks," IEEE Trans. Microw. Theory Techn., vol. 64, no. 12, pp. 1-16, Dec., 2016.

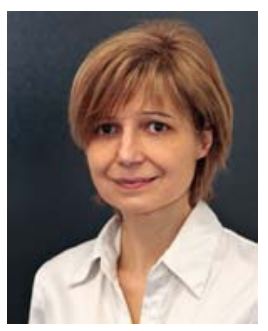

Almudena Suárez (M'96-SM'01-F'12) was born in Santander, Spain. She received the Licentiate degree in Electronic Physics and the Ph.D. degree from the University of Cantabria, Santander, Spain, in 1987 and 1992, respectively, and the Ph.D. degree in Electronics from the University of Limoges, Limoges, France, in 1993. She is currently a full professor at University of Cantabria (Spain) and head of the research group Microwave Engineering and Radiocommunication Systems. She is a Fellow member of the IEEE (Institute of Electrical and Electronic Engineers, New Jersey, USA). She was also an IEEE Distinguished Microwave Lecturer during the period 2006-2008. She has authored the book Analysis and design of autonomous microwave circuits (IEEE-Wiley, 2009) and co-authored the book Stability analysis of nonlinear microwave circuit' (Artech House, 2003).

Prof. Suárez is a member of the technical committees of IEEE International Microwave Symposium (IEEE MTT-S) and the European Microwave Week. She is a member of the Board of Directors of European Microwave Association. She is an associate editor of IEEE Microwave Magazine. She was the coordinator of the Communications and Electronic Technology Area for the Spanish National Evaluation and Foresight Agency (ANEP) between 2009 and 2013. She was the chair of the 2014 and 2015 editions of IEEE Topical Conference on RF/Microwave Power Amplifiers (PAWR), in Newport Beach and San Diego. She was the General TPC Chair of European Microwave Wekk 2018. Prof. Suárez was the Editor-in-Chief of the International Journal of 
Microwave and Wireless Technologies from Cambridge University Press journals from 2013 to 2018 and is currently an Associate Editor for IEEE Microwave Magazine and the IEEE Transactions on Microwave Theory and Techniques.

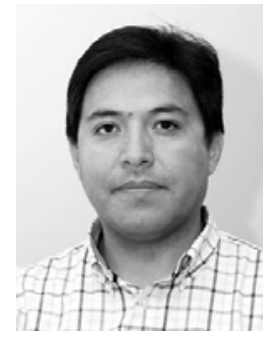

Franco Ramírez (S'03-A'05-M'05-SM'16) obtained the Licentiate degree in electronic systems engineering degree from the Military School of Engineering (EMI) in La Paz, Bolivia, in 2000 and the Ph.D. degree in Communications Engineering from the University of Cantabria, Santander, Spain in 2005. From 1999 to 2000, he worked for Ericsson de Bolivia Telecomunicaciones, where he was involved in projects related with GSM and TDMA technologies. From 2009 to 2013, Dr. Ramírez was a Research Fellow of the "Ramón y Cajal" Programme, funded by the Spanish Ministry of Science and Innovation, at the Communications Engineering Department of the University of Cantabria, where he is currently an Associate Professor. His research interests include phase noise, stability and the development of nonlinear techniques for the analysis and design of autonomous microwave circuits. 